Article

\title{
Some Results in Weak Pseudo-Quasi-Wajsberg Algebras
}

\author{
Wenjun Liu and Wenjuan Chen *(1) \\ School of Mathematical Sciences, University of Jinan, No. 336, West Road of Nan Xinzhuang, Jinan 250022, China; \\ liuwenjun@mail.ujn.edu.cn \\ * Correspondence: wjchenmath@gmail.com
}

Received: 26 January 2019; Accepted: 23 March 2019; Published: 28 March 2019

check for updates

\begin{abstract}
In this paper we continue to investigate weak pseudo-quasi-Wajsberg algebras which are called weak PQW-algebras for short. First, some definitions are recalled and the basic properties of weak PQW-algebras are presented. Next, we define the notions of type-I filters, type-II filters, left weak filters and right weak filters of a weak PQW-algebra and study related properties of them. We also discuss the relation between normal filters and filter congruences on a weak PQW-algebra. Finally, we characterize the relationship between weak PQW-algebras and some bounded residuated quasi-ordered monoids with supplementary conditions.
\end{abstract}

Keywords: weak pseudo-quasi-Wajsberg algebras; filters; weak filters; residuated

2010 Mathematics Subject Classification: 03G27; 06B10; 08C15

\section{Introduction}

In recent years, various kinds of non-classical algebras as the semantical systems of non-classical logic systems have been extensively introduced and studied, for example, MV-algebras [1], BL-algebras [2], MTL-algebras [3], BCK-algebras [4], BCI-algebras [5] and their non-commutative cases [6-11]. Among these logical algebras, MV-algebras and their non-commutative cases (pseudo-MV algebras) are the most widely studied algebras. Since people are interested in the logical aspects of this subject, it makes sense to redefine the algebras in such a way that implication is a primitive operation. Thus Wajsberg algebras were defined and investigated in [12] and authors showed that Wajsberg algebras and MV-algebras were equivalent. Subsequently, pseudo-Wajsberg algebras were introduced by Ceterchi, which were equivalent to pseudo-MV algebras [13]. For any pseudo-Wajsberg algebra, if two implications coincide, then it is a Wajsberg algebra. Hence pseudo-Wajsberg algebras are the generalization of Wajsberg algebras. To generalize pseudo-Wajsberg algebras and pseudo-MV algebras, Ceterchi introduced and studied weak pseudo-Wajsberg algebras in [14]. She divided the axiom $(a \rightarrow b) \rightsquigarrow b=(b \rightarrow a) \rightsquigarrow a=(a \rightsquigarrow b) \rightarrow b=$ $(b \leadsto a) \rightarrow a$ into two parts in order to obtain two orders and two lattice structures on the weakened structures.

Recently, quantum computational logic, which is based on the theory of quantum computation has been developed. This new form logic, unlike orthodox quantum logic, is much closer to "fuzzy logic" [15]. To study it, Ledda et al. introduced quasi-MV algebra as a first significant step towards the algebraic characterization of the quantum computational logic [16]. From pure algebraic point, quasi-MV algebras can be regarded as the another generalization of MV-algebras (in which 0 is no longer a neutral element of the operation sum). In 2016, pseudo-quasi-MV algebras were introduced in $[17,18]$ as a non-commutative generalization of quasi-MV algebras. Meanwhile, pseudo-quasi-Wajsberg algebras were also defined and 
investigated for the equivalence to pseudo-quasi-MV algebras in [18]. Following Ceterchi's way, Liu et al. introduced the concepts of weak pseudo-quasi-Wajsberg algebras (weak PQW-algebras, for short) and study some related properties of them in [19].

To find more connections between weak PQW-algebras and other algebraic structures, we want to continue the investigation on weak PQW-algebras in this paper. It is well known that the basic tool for the investigation of algebraic structures is filter theory [7,20-23]. On the one hand, the filters in connection with congruences on algebras are deeply investigated. On the other hand, filters are same to the notions of deductive systems which correspond sets of provable formulas from the logical point of view. Hence the theory of filters play an important role in the study of logical algebras and then we focus on discussing the filter theory of weak PQW-algebras. The paper is organized as follows. In Section 2, we recall some definitions and results which will be used in what follows. In Section 3, we introduce two types of filters and left/right weak filters in a weak PQW-algebra and some properties of them are investigated. We also discuss the connection between normal filters and filter congruences on a weak PQW-algebra. In Section 4, we discuss the relationship between weak PQW-algebras and some bounded residuated quasi-ordered monoids with supplementary conditions.

\section{Weak Pseudo-Quasi-Wajsberg Algebras}

In this section, we recall the definitions of Wajsberg algebras, pseudo-Wajsberg algebras, quasi-Wajsberg algebras and weak pseudo-Wajsberg algebras. Parallelly, we list the definitions of MV-algebras, pseudo-MV algebras and quasi-MV algebras. Some basic properties of weak pseudo-quasi-Wajsberg algebras are also presented.

Definition 1. [12] Let $A=\left\langle A ; \rightarrow,{ }^{\prime}, 1\right\rangle$ be an algebra of type $\langle 2,1,0\rangle$. Then it is called a Wajsberg algebra, if the following axioms are satisfied for all $a, b, c \in A$ :

(W1) $1 \rightarrow a=a$;

$($ W2) $(a \rightarrow b) \rightarrow((b \rightarrow c) \rightarrow(a \rightarrow c))=1$;

(W3) $(a \rightarrow b) \rightarrow b=(b \rightarrow a) \rightarrow a$;

(W4) $\left(a^{\prime} \rightarrow b^{\prime}\right) \rightarrow(b \rightarrow a)=1$.

Wajsberg algebras were equivalent to the following MV-algebras.

Definition 2. [1] Let $A=\left\langle A ; \oplus,{ }^{\prime}, 0\right\rangle$ be an algebra of type $\langle 2,1,0\rangle$. Then it is called an $M V$-algebra, if the following axioms are satisfied for all $a, b, c \in A$ :

$(M V 1) a \oplus(b \oplus c)=(a \oplus b) \oplus c$

(MV2) $a \oplus b=b \oplus a$;

(MV3) $a \oplus 0=a$;

$(M V 4) a^{\prime \prime}=a$;

$(M V 5) a \oplus 0^{\prime}=0^{\prime}$;

$(M V 6)\left(a^{\prime} \oplus b\right)^{\prime} \oplus b=\left(b^{\prime} \oplus a\right)^{\prime} \oplus a$.

Ceterchi generalized Wajsberg algebras to non-commutative cases and introduced pseudo-Wajsberg algebras in [13] which were equivalent to pseudo-MV algebras.

Definition 3. [13] Let $A=\left\langle A ; \rightarrow, \rightsquigarrow,^{-}, \sim, 1\right\rangle$ be an algebra of type $\langle 2,2,1,1,0\rangle$. Then it is called a pseudo-Wajsberg algebra, if the following axioms are satisfied for all $a, b, c \in A$ : 
(PW1) $1 \rightarrow a=a=1 \leadsto a$;

(PW2) $(a \rightarrow b) \rightsquigarrow b=(b \rightarrow a) \rightsquigarrow a=(a \rightsquigarrow b) \rightarrow b=(b \rightsquigarrow a) \rightarrow a$;

(PW3) $(a \rightarrow b) \rightarrow((b \rightarrow c) \rightsquigarrow(a \rightarrow c))=1$,

$(a \rightsquigarrow b) \rightsquigarrow((b \rightsquigarrow c) \rightarrow(a \rightsquigarrow c))=1$;

$\left(\right.$ PW4) $\left(a^{\sim} \rightarrow b^{\sim}\right) \rightarrow(b \rightsquigarrow a)=1,\left(a^{-} \rightsquigarrow b^{-}\right) \rightarrow(b \rightarrow a)=1$;

(PW5) $\left(a \rightarrow b^{-}\right)^{\sim}=\left(b \rightsquigarrow a^{\sim}\right)^{-}$.

Definition 4. [10] Let $A=\left\langle A ; \oplus^{-}, \sim, 1\right\rangle$ be an algebra of type $\langle 2,1,1,0\rangle$. Then it is called a pseudo-MV algebra, if the following axioms are satisfied for all $a, b, c \in A$ :

(PMV1) $1^{-}=1^{\sim}$;

$(P M V 2) a \oplus(b \oplus c)=(a \oplus b) \oplus c$

$(P M V 3) a \oplus 1^{-}=1^{-} \oplus a=a$;

$(P M V 4) a \oplus 1=1 \oplus a=1$;

(PMV5) $\left(a^{-} \oplus b^{-}\right)^{\sim}=\left(a^{\sim} \oplus b^{\sim}\right)^{-}$;

$\left(\right.$ PMV6) $a \oplus\left(a^{\sim} \odot b\right)=b \oplus\left(b^{\sim} \odot a\right)=\left(a \odot b^{-}\right) \oplus b=\left(b \odot a^{-}\right) \oplus a ;$

$\left(\right.$ PMV7) $a \odot\left(a^{-} \oplus b\right)=\left(a \oplus b^{\sim}\right) \odot b$;

$\left(\right.$ PMV8) $a^{-\sim}=a$,

where $b \odot a=\left(a^{-} \oplus b^{-}\right)^{\sim}$.

Further, Ceterchi introduced weak pseudo-Wajsberg algebras as the generalization of pseudo-Wajsberg algebras in [14]. She divided the axiom (PW2) into two parts in order to obtain two orders and two lattice structures on the weakened structures.

Definition 5. [14] Let $A=\langle A ; \rightarrow, \rightsquigarrow, 0,1\rangle$ be an algebra of type $\langle 2,2,0,0\rangle$. Then it is called a weak pseudo-Wajsberg algebra, if the following axioms are satisfied for all $a, b, c \in A$ :

$(w P W 1) 1 \rightarrow a=a=1 \rightsquigarrow a$;

$(w P W 2)(a \rightarrow b) \rightsquigarrow b=(b \rightarrow a) \rightsquigarrow a,(a \rightsquigarrow b) \rightarrow b=(b \rightsquigarrow a) \rightarrow a$;

$(w P W 3)(a \rightarrow b) \rightarrow((b \rightarrow c) \rightsquigarrow(a \rightarrow c))=1$,

$(a \rightsquigarrow b) \rightsquigarrow((b \rightsquigarrow c) \rightarrow(a \rightsquigarrow c)=1 ;$

(wPW4) $0 \rightarrow a=0 \rightsquigarrow a=1$;

$(w P W 5) a \rightarrow(b \rightsquigarrow c)=b \rightsquigarrow(a \rightarrow c)$.

On any weak pseudo-Wajsberg algebra, one can define $a^{-}=a \rightarrow 0$ and $a^{\sim}=a \rightsquigarrow 0$ for all $a \in A$.

Proposition 1. [14] Let A be a weak pseudo-Wajsberg algebra. If it satisfies

(PW3) $(a \rightsquigarrow b) \rightarrow b=(b \rightsquigarrow a) \rightarrow a=(b \rightarrow a) \rightsquigarrow a=(a \rightarrow b) \rightsquigarrow b$;

(PW5) $\left(a \rightarrow b^{-}\right)^{\sim}=\left(b \rightsquigarrow a^{\sim}\right)^{-}$,

then it is a pseudo-Wajsberg algebra.

On the other hand, quasi-Wajsberg algebras were also introduced as a generalization of Wajsberg algebras. Any quasi-Wajsberg algebra is with the condition $1 \rightarrow a=a$, then it is a Wajsberg algebra.

Definition 6. [20] Let $A=\langle A ; \rightarrow,, 1\rangle$ be an algebra of type $\langle 2,1,0\rangle$. Then it is called a quasi-Wajsberg algebra, if the following axioms are satisfied for all $a, b, c \in A$ : 
$(Q W 1) 1 \rightarrow(a \rightarrow b)=a \rightarrow b$;

$(\mathrm{QW} 2)(a \rightarrow b) \rightarrow((b \rightarrow c) \rightarrow(a \rightarrow c))=1$;

(QW3) $(a \rightarrow b) \rightarrow b=(b \rightarrow a) \rightarrow a$;

$\left(\right.$ QW4) $\left(a^{\prime} \rightarrow b^{\prime}\right) \rightarrow(b \rightarrow a)=1$;

(QW5) $a^{\prime \prime}=a$;

$(Q W 6) 1 \rightarrow(1 \rightarrow a)^{\prime}=(1 \rightarrow a)^{\prime}$.

Indeed, any quasi-Wajsberg algebra was equivalent to a quasi-MV algebra [16].

Definition 7. [16] Let $A=\left\langle A ; \oplus,{ }^{\prime}, 0\right\rangle$ be an algebra of type $\langle 2,1,0\rangle$. Then it is called a quasi-MV algebra, if the following axioms are satisfied for all $a, b, c \in A$ :

$(Q M V 1) a \oplus(b \oplus c)=(a \oplus c) \oplus b ;$

$(Q M V 2) a^{\prime \prime}=a$;

$(Q M V 3) a \oplus 0^{\prime}=0^{\prime}$;

(QMV4) $\left(a^{\prime} \oplus b\right)^{\prime} \oplus b=\left(b^{\prime} \oplus a\right)^{\prime} \oplus a ;$

$(Q M V 5)(a \oplus 0)^{\prime}=a^{\prime} \oplus 0$;

$(Q M V 6)(a \oplus b) \oplus 0=a \oplus b$.

In [18], Liu et al. generalized quasi-Wajsberg algebras to non-commutative cases and introduced pseudo-quasi-Wajsberg algebras. They also showed that pseudo-quasi-Wajsberg algebras were equivalent to pseudo-quasi-MV algebras.

Definition 8. [18] Let $A=\left\langle A ; \rightarrow, \rightsquigarrow,^{\prime}, 1\right\rangle$ be an algebra of type $\langle 2,2,1,0\rangle$. Then it is called a pseudo-quasi-Wajsberg algebra, if the following axioms are satisfied for all $a, b, c \in A$ :

$(P Q W 1) 1 \rightarrow a=1 \rightsquigarrow a$;

$(P Q W 2) 1 \rightarrow(a \rightarrow y)=a \rightarrow b, 1 \rightsquigarrow(a \rightsquigarrow b)=a \rightsquigarrow b$;

(PQW3) $(a \rightsquigarrow b) \rightarrow b=(b \rightsquigarrow a) \rightarrow a=(b \rightarrow a) \rightsquigarrow a=(a \rightarrow b) \rightsquigarrow b$;

$(P Q W 4)(a \rightarrow b) \rightarrow((b \rightarrow c) \rightsquigarrow(a \rightarrow c))=1$,

$(a \rightsquigarrow b) \rightsquigarrow((b \rightsquigarrow c) \rightarrow(a \rightsquigarrow c))=1 ;$

$\left(\right.$ PQW5) $\left(a^{\prime} \rightsquigarrow b^{\prime}\right) \rightarrow(b \rightarrow a)=1,\left(a^{\prime} \rightarrow b^{\prime}\right) \rightarrow(b \rightsquigarrow a)=1$;

$(P Q W 6) a \rightarrow b^{\prime}=b \leadsto a^{\prime}$;

$(P Q W 7) 1 \rightarrow(1 \rightarrow a)^{\prime}=(1 \rightarrow a)^{\prime}, 1 \rightsquigarrow(1 \rightsquigarrow a)^{\prime}=(1 \rightsquigarrow a)^{\prime}$;

$(P Q W 8) a^{\prime \prime}=a$.

Based on pseudo-quasi-Wajsberg algebras, authors introduced the concepts of weak pseudo-quasiWajsberg algebras following Ceterchi's way and studied the related properties of them in [19].

Definition 9. [19] Let $A=\langle A ; \rightarrow, \rightsquigarrow,, 1\rangle$ be an algebra of type $\langle 2,2,1,0\rangle$. Then it is called a weak pseudo-quasi-Wajsberg algebra (weak $P Q W$-algebra, for short), if the following axioms are satisfied for all $a, b, c \in A$ :

$(w P Q W 1) 1 \rightarrow a=1 \rightsquigarrow a$;

$(w P Q W 2) 1 \rightarrow(a \rightarrow b)=a \rightarrow b, 1 \rightsquigarrow(a \rightsquigarrow b)=a \rightsquigarrow b$;

(wPQW3) $(a \rightarrow b) \rightsquigarrow b=(b \rightarrow a) \rightsquigarrow a$,

$(a \rightsquigarrow b) \rightarrow b=(b \rightsquigarrow a) \rightarrow a$;

$(w P Q W 4)(a \rightarrow b) \rightarrow((b \rightarrow c) \rightsquigarrow(a \rightarrow c))=1$,

$(a \rightsquigarrow b) \rightsquigarrow((b \rightsquigarrow c) \rightarrow(a \rightsquigarrow c)=1 ;$

$(w P Q W 5)\left(a^{\prime} \rightsquigarrow b^{\prime}\right) \rightarrow(b \rightarrow a)=1,\left(a^{\prime} \rightarrow b^{\prime}\right) \rightarrow(b \rightsquigarrow a)=1$ 
$(w P Q W 6) a \rightarrow b^{\prime}=b \leadsto a^{\prime}$

$(w P Q W 7) 1 \rightarrow(1 \rightarrow a)^{\prime}=(1 \rightarrow a)^{\prime}$;

(wPQW8) $a^{\prime \prime}=a ;$

$(w P Q W 9) a \rightarrow(b \rightsquigarrow c)=b \rightsquigarrow(a \rightarrow c)$.

Obviously, any pseudo-quasi-Wajsberg algebra is a weak PQW-algebra. Conversely, any weak PQW-algebra which satisfies the axiom $(a \rightsquigarrow b) \rightarrow b=(b \rightsquigarrow a) \rightarrow a=(b \rightarrow a) \rightsquigarrow a=(a \rightarrow b) \rightsquigarrow b$ is a pseudo-quasi-Wajsberg algebra.

Below we list some properties of weak PQW-algebras. The proofs can be seen in [19].

Proposition 2. Let $A=\left\langle A ; \rightarrow, \rightsquigarrow,{ }^{\prime}, 1\right\rangle$ be a weak $P Q W$-algebra. Then the following results hold for all $a, b, c \in A$ :

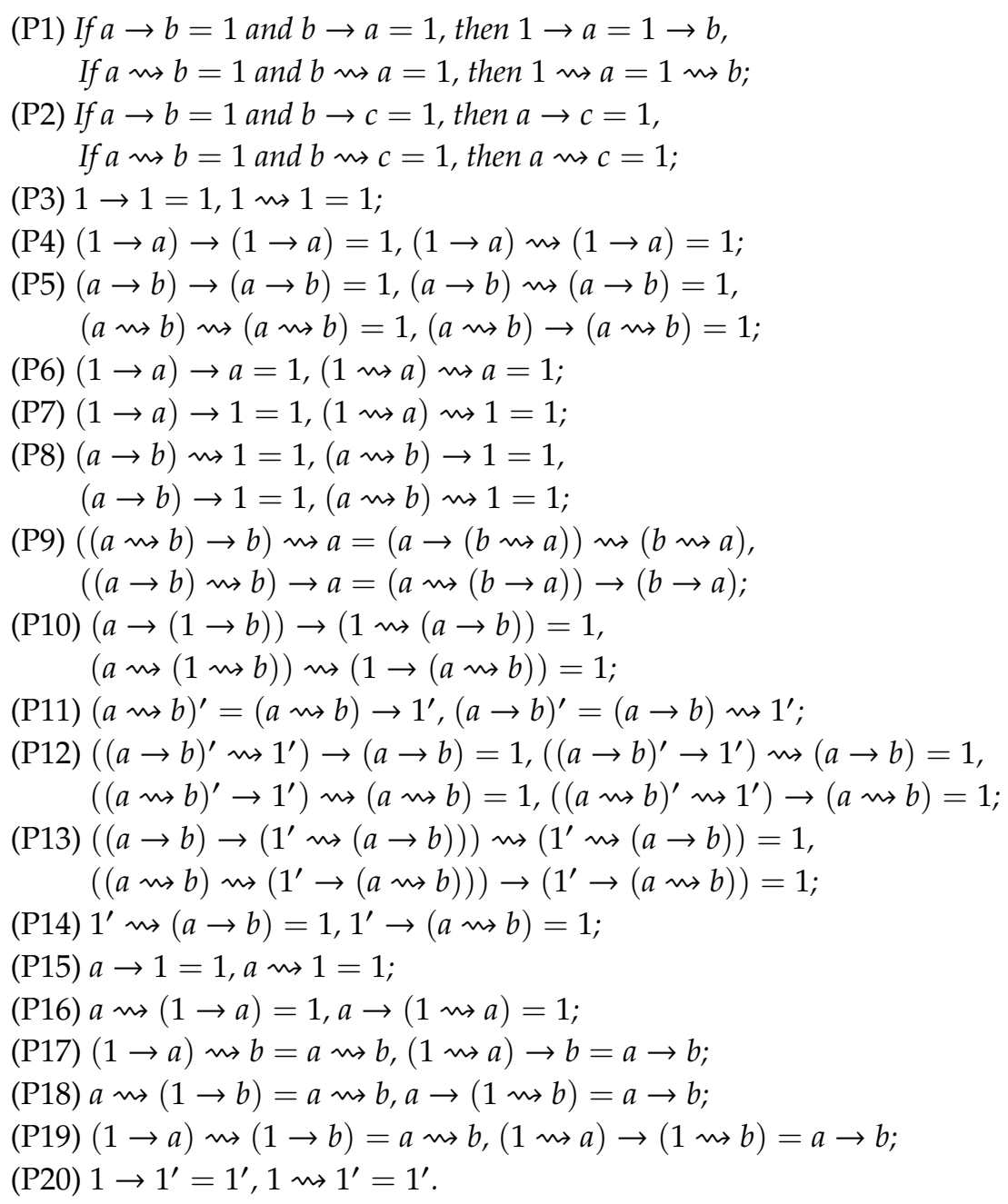

Remark 1. Here, some additional explanations are required. The axiom (wPQW6) in Definition 5 can be implied by other axioms. By (wPQW5) and (wPQW8), we have $\left(b \rightsquigarrow a^{\prime}\right) \rightarrow\left(a \rightarrow b^{\prime}\right)=1$ and $\left(a \rightarrow b^{\prime}\right) \rightarrow\left(b \rightsquigarrow a^{\prime}\right)=1$ for any $a, b \in A$, so $1 \rightarrow\left(b \rightsquigarrow a^{\prime}\right)=1 \rightarrow\left(a \rightarrow b^{\prime}\right)$ by (P1). Using (wPQW2) and (wPQW1), we get $a \rightarrow b^{\prime}=b \rightsquigarrow a^{\prime}$. Hence in the following we omit the axiom (wPQW6) and renumber the axioms in Definition 5. 
On a weak PQW-algebra, one can define two relations $a \leq_{1} b$ iff $a \rightarrow b=1$ and $a \leq_{2} b$ iff $a \rightsquigarrow b=1$. Let us mention that on any pseudo-quasi-Wajsberg algebra, it easily gets that $a \rightarrow b=1$ iff $a \rightsquigarrow b=1$. Thus the relations $\leq_{1}$ and $\leq_{2}$ coincide in any pseudo-quasi-Wajsberg algebra.

Suppose that $A$ is a weak PQW-algebra. Then the relations $\leq_{1}$ and $\leq_{2}$ are reflexivity and transitivity. However, they are not antisymmetry. In fact, if $a \leq_{1} b$ and $b \leq_{1} a$, then $1 \rightarrow a=1 \rightarrow b$ and if $a \leq_{2} b$ and $b \leq_{2} a$, then $1 \rightsquigarrow a=1 \rightsquigarrow b$. In general, we may not get $a=b$. We denote $R(A)=\{a \in A \mid 1 \rightarrow a=a\}$. Then the relations $\leq_{1}$ and $\leq_{2}$ restricted to $R(A)$ are antisymmetry, so they are partial orderings on $R(A)$.

Proposition 3. Let $A$ be a weak $P Q W$-algebra. Then the following are true for all $a, b, c \in A$ :

(P21) $a \leq_{1} 1 \rightsquigarrow a$ and $a \leq_{2} 1 \rightarrow a$;

(P22) $1 \rightsquigarrow a \leq_{1}$ and $1 \rightarrow a \leq_{2} a$;

(P23) $a \rightarrow b \leq_{1}(b \rightarrow c) \rightsquigarrow(a \rightarrow c), a \rightsquigarrow b \leq_{2}(b \rightsquigarrow c) \rightarrow(a \rightsquigarrow c)$;

$(\mathrm{P} 24) a \rightsquigarrow b \leq_{1}(c \rightsquigarrow a) \rightsquigarrow(c \rightsquigarrow b), a \rightarrow b \leq_{2}(c \rightarrow a) \rightarrow(c \rightarrow b)$;

(P25) $a \leq_{1}(b \leadsto a), a \leq_{2}(b \rightarrow a)$;

(P26) $a \leq_{1} b \leadsto c \Leftrightarrow b \leq_{2} a \rightarrow c, a \leq_{2} b \rightarrow c \Leftrightarrow b \leq_{1} a \rightsquigarrow c$;

(P27) $a \leq_{1} b \Rightarrow b \rightarrow c \leq_{2} a \rightarrow c, a \leq_{2} b \Rightarrow b \rightsquigarrow c \leq_{1} a \rightsquigarrow c$;

(P28) $a \leq_{1} b \Rightarrow c \rightarrow a \leq_{1} c \rightarrow b, a \leq_{2} b \Rightarrow c \rightsquigarrow a \leq_{2} c \rightsquigarrow b$;

(P29) $a \leq_{1} b \Leftrightarrow b^{\prime} \leq_{2} a^{\prime}, a \leq_{2} b \Leftrightarrow b^{\prime} \leq_{1} a^{\prime}$.

We can also define some binary operations as follows: $a \vee_{1} b=(a \rightarrow b) \rightsquigarrow b=(b \rightarrow a) \rightsquigarrow a$, $a \vee_{2} b=(a \rightsquigarrow b) \rightarrow b=(b \rightsquigarrow a) \rightarrow a, a \wedge_{1} b=\left(a^{\prime} \vee_{2} b^{\prime}\right)^{\prime}$ and $a \wedge_{2} b=\left(a^{\prime} \vee_{1} b^{\prime}\right)^{\prime}$

Proposition 4. Let $A$ be a weak $P Q W$-algebra. Then the following are true for all $a, b \in A$ :

(1) $a \vee_{1} b$ is a supremum and $a \wedge_{1} b$ is an infimum for $a$ and $b$ with respect to $\leq_{1}$;

(2) $a \vee_{2} b$ is a supremum and $a \wedge_{2} b$ is an infimum for $a$ and $b$ with respect to $\leq_{2}$.

Proposition 5. Let $A$ be a weak $P Q W$-algebra. Then the following are true for all $a, b \in A$ :

(P30) $a \leq_{1} b \Leftrightarrow a \vee_{1} b=1 \rightarrow b$;

(P31) $a \leq_{2} b \Leftrightarrow a \vee_{2} b=1 \leadsto b$;

(P32) $a \leq_{1} b \Leftrightarrow a \wedge_{1} b=1 \rightarrow a$;

(P33) $a \leq_{2} b \Leftrightarrow a \wedge_{2} b=1 \leadsto a$;

(P34) $a \vee_{1} b=b \vee_{1}$ a and $a \wedge_{1} b=b \wedge_{1} a$;

(P35) $\left(a \vee_{1} b\right) \vee_{1} c=a \vee_{1}\left(b \vee_{1} c\right)$ and $\left(a \wedge_{1} b\right) \wedge_{1} c=a \wedge_{1}\left(b \wedge_{1} c\right)$;

(P36) $a \vee_{1} a=1 \rightarrow$ a and $a \wedge_{1} a=1 \rightarrow a$;

(P37) $\left(a \vee_{1} b\right) \wedge_{1} a=1 \rightarrow a$ and $\left(a \wedge_{1} b\right) \vee_{1} a=1 \rightarrow a$;

(P38) $a \vee_{2} b=b \vee_{2} a$ and $a \wedge_{2} b=b \wedge_{2} a$;

(P39) $\left(a \vee_{2} b\right) \vee_{2} c=a \vee_{2}\left(b \vee_{2} c\right)$ and $\left(a \wedge_{2} b\right) \wedge_{2} c=a \wedge_{2}\left(b \wedge_{2} c\right)$;

(P40) $a \vee_{2} a=1 \rightsquigarrow a$ and $a \wedge_{2} a=1 \rightsquigarrow a$;

(P41) $\left(a \vee_{2} b\right) \wedge_{2} a=1 \rightsquigarrow a$ and $\left(a \wedge_{2} b\right) \vee_{1} a=1 \rightsquigarrow a$;

(P42) $\left(a \vee_{1} b\right) \rightarrow c=(a \rightarrow c) \wedge_{2}(b \rightarrow c),\left(a \vee_{2} b\right) \rightsquigarrow c=(a \rightsquigarrow c) \wedge_{1}(b \rightsquigarrow c)$;

$(\mathrm{P} 43) c \rightarrow\left(a \wedge_{1} b\right)=(c \rightarrow a) \wedge_{1}(c \rightarrow b), c \rightsquigarrow\left(a \wedge_{2} b\right)=(c \rightsquigarrow a) \wedge_{2}(c \rightsquigarrow b)$;

(P44) $\left(a \vee_{1} b\right) \rightarrow b=a \rightarrow b,\left(a \vee_{2} b\right) \rightsquigarrow b=a \rightsquigarrow b$;

(P45) $a \rightarrow\left(a \wedge_{1} b\right)=a \rightarrow b, a \rightsquigarrow\left(a \wedge_{2} b\right)=a \rightsquigarrow b$. 
Like the relationship between weak pseudo-MV algebras and weak pseudo-Wajsberg algebras was introduced in [14], we defined weak pseudo-quasi-MV algebras and showed that there exists a corresponded relation between weak PQW-algebras and weak pseudo-quasi-MV algebras.

Definition 10. [19] Let $A=\left\langle A ; \oplus^{\prime}, 0\right\rangle$ be an algebra of type $\langle 2,1,0\rangle$. Then it is called a weak pseudo-quasi-MV algebra, if the following axioms are satisfied for all $a, b, c \in A$ :

$(w P Q M V 1)(a \oplus b) \oplus c=a \oplus(b \oplus c)$;

$(w P Q M V 2) a \oplus b \oplus 0=a \oplus b$;

$(w P Q M V 3) a \oplus 0=0 \oplus a$;

$(w P Q M V 4) a \oplus 0^{\prime}=0^{\prime}=0^{\prime} \oplus a ;$

$(w P Q M V 5)(a \oplus 0)^{\prime}=a^{\prime} \oplus 0$;

$(w P Q M V 6) b \oplus\left(a^{\prime} \oplus b\right)^{\prime}=a \oplus\left(b^{\prime} \oplus a\right)^{\prime},\left(b \oplus a^{\prime}\right)^{\prime} \oplus b=\left(a \oplus b^{\prime}\right)^{\prime} \oplus a ;$

$(w P Q M V 7) a^{\prime \prime}=a$.

The following results were proved in [19].

Theorem 1. Let $A=\left\langle A ; \oplus,^{\prime}, 0\right\rangle$ be a weak pseudo-quasi-MV algebra. If we define the binary operations $a \rightarrow b=a^{\prime} \oplus b, a \rightsquigarrow b=b \oplus a^{\prime}$ and a constant $1=0^{\prime}$, then $f(A)=\left\langle A ; \rightarrow, \rightsquigarrow,^{\prime}, 1\right\rangle$ is a weak PQW-algebra.

Conversely, we have

Theorem 2. Let $A=\left\langle A ; \rightarrow, \rightsquigarrow,^{\prime}, 1\right\rangle$ be a weak PQW-algebra. If we define the binary operation $a \oplus b=a^{\prime} \rightarrow b=$ $b^{\prime} \leadsto a$ and a constant $0=1^{\prime}$, then $g(A)=\left\langle A ; \oplus^{\prime}, 0\right\rangle$ is a weak pseudo-quasi-MV algebra.

\section{Filters, Weak Filters and Homomorphisms}

In this section, we introduce and study filters and weak filters of weak PQW-algebras. Based on the induced relations $\leq_{1}$ and $\leq_{2}$, we can define type-I/type-II filters and then generalize them to left/right weak filters. The properties of them under the homomorphisms are also discussed.

Suppose that $A$ is a weak PQW-algebra. We can define binary operations as follows: $a \oplus b=a^{\prime} \rightarrow b=$ $b^{\prime} \rightsquigarrow a$ and $a \cdot b=\left(b^{\prime} \oplus a^{\prime}\right)^{\prime}$. Then $a \cdot b=\left(b \rightarrow a^{\prime}\right)^{\prime}=\left(a \rightsquigarrow b^{\prime}\right)^{\prime}$.

Lemma 1. Let $A$ be a weak $P Q W-a l g e b r a$. Then

(1) For all $a, b \in A$, we have $a \leq_{1} a \oplus b$ and $b \leq_{2} a \oplus b$;

(2) For all $a, b \in A$, we have $a \cdot b \leq_{1}$ a and $a \cdot b \leq_{2} b$.

Proof. (1) Since $a \rightarrow(a \oplus b)=a \rightarrow\left(b^{\prime} \rightsquigarrow a\right)=b^{\prime} \rightsquigarrow(a \rightarrow a)=b^{\prime} \rightsquigarrow 1=1$ by (wPQW9) and (P15), we have $a \leq_{1} a \oplus b$. Now, we calculate $b \leadsto(a \oplus b)=b \leadsto\left(a^{\prime} \rightarrow b\right)=a^{\prime} \rightarrow(b \leadsto b)=a^{\prime} \rightarrow 1=1$, thus $b \leq_{2} a \oplus b$.

(2) Since $(a \cdot b) \rightarrow a=\left(b \rightarrow a^{\prime}\right)^{\prime} \rightarrow a=a^{\prime} \rightsquigarrow\left(b \rightarrow a^{\prime}\right)=b \rightarrow\left(a^{\prime} \rightsquigarrow a^{\prime}\right)=b \rightarrow 1=1$, we have $a \cdot b \leq_{1} a$. On the other hand, we have $(a \cdot b) \rightsquigarrow b=\left(a^{\prime} \leadsto b\right)^{\prime} \rightsquigarrow b=b^{\prime} \rightarrow\left(a^{\prime} \rightsquigarrow b\right)=a^{\prime} \rightsquigarrow(b \rightarrow b)=$ $a^{\prime} \leadsto 1=1$, thus $a \cdot b \leq_{2} b$.

Lemma 2. Let $A$ be a weak $P Q W$-algebra. For all $a, b, x \in A$ we have:

(1) If $a \leq_{1} b$, then $x \oplus a \leq_{1} x \oplus b$ and if $a \leq_{2} b$, then $a \oplus x \leq_{2} b \oplus x$;

(2) If $a \leq_{1} b$, then $x \cdot a \leq_{1} x \cdot b$ and if $a \leq_{2} b$, then $a \cdot x \leq_{2} b \cdot x$. 
Proof. (1) Suppose that $a \leq_{1} b$, then by (P28) we have $x^{\prime} \rightarrow a \leq_{1} x^{\prime} \rightarrow b$, it follows that $x \oplus a \leq_{1} x \oplus b$. Assume that $a \leq_{2} b$, then $b^{\prime} \leq_{1} a^{\prime}$. Using (P27), we have $a^{\prime} \rightarrow x \leq_{2} b^{\prime} \rightarrow x$, so $a \oplus x \leq_{2} b \oplus x$.

(2) Suppose that $a \leq_{1} b$, we have $b^{\prime} \leq_{2} a^{\prime}$, it follows that $b^{\prime} \oplus x^{\prime} \leq_{2} a^{\prime} \oplus x^{\prime}$ by (1), then $\left(a^{\prime} \oplus x^{\prime}\right)^{\prime} \leq_{1}$ $\left(b^{\prime} \oplus x^{\prime}\right)^{\prime}$, so $x \cdot a \leq_{1} x \cdot b$. The other can be proved analogously.

Now we give the definition and equivalent characterization of type-I filter.

Definition 11. Let $A$ be a weak $P Q W$-algebra and $F$ be a non-empty subset of $A$. Then $F$ is called a type-I filter of $A$, if it satisfies the following conditions:

(FI1) If $a, b \in F$, then $a \cdot b \in F$;

(FI2) If $a \in F$ and $b \in A$ with $a \leq_{1} b$, then $b \in F$.

Proposition 6. Let $A$ be a weak $P Q W-a l g e b r a$ and $F$ be a subset of $A$. Then the following conditions are equivalent:

(1) $F$ is a type-I filter of $A$;

(2) $1 \in F$ and if $a \in F$ and $a \rightarrow b \in F$, then $b \in F$.

Proof. (1) $\Rightarrow(2)$ For all $a \in F$, since $a \leq_{1} 1$, we have $1 \in F$. Assume that $a \in F$ and $a \rightarrow b \in F$. Then $a \cdot(a \rightarrow b) \in F$ and we calculate $(a \cdot(a \rightarrow b)) \rightarrow b=\left((a \rightarrow b) \rightarrow a^{\prime}\right)^{\prime} \rightarrow b=b^{\prime} \rightsquigarrow\left((a \rightarrow b) \rightarrow a^{\prime}\right)=(a \rightarrow$ $b) \rightarrow\left(b^{\prime} \leadsto a^{\prime}\right)=(a \rightarrow b) \rightarrow(a \rightarrow b)=1$, it follows that $a \cdot(a \rightarrow b) \leq_{1} b$, so $b \in F$.

$(2) \Rightarrow(1)$ Let $a, b \in F$. Then $b \rightarrow(a \rightarrow(a \cdot b))=b \rightarrow\left[a \rightarrow\left(b \rightarrow a^{\prime}\right)^{\prime}\right]=b \rightarrow\left[\left(b \rightarrow a^{\prime}\right) \rightsquigarrow a^{\prime}\right]=(b \rightarrow$ $\left.a^{\prime}\right) \rightsquigarrow\left(b \rightarrow a^{\prime}\right)=1$, so $b \rightarrow(a \rightarrow a \cdot b) \in F$. Since $b \in F$, we have $a \rightarrow(a \cdot b) \in F$. Similarly, because $a \in F$, we get $a \cdot b \in F$. Moreover, if $a \in F$ and $b \in A$ with $a \leq_{1} b$, then $a \rightarrow b=1 \in F$, it turns out that $b \in F$. Thus $F$ is a type-I filter of $A$.

Below we present the definition of type-II filter.

Definition 12. Let $A$ be a weak $P Q W$-algebra and $F$ be a non-empty subset of $A$. Then $F$ is called a type-II filter of $A$, if it satisfies the following conditions:

(FII1) If $a, b \in F$, then $a \cdot b \in F$;

(FII2) If $a \in F$ and $b \in A$ with $a \leq_{2} b$, then $b \in F$.

Proposition 7. Let $A$ be a weak $P Q W-a l g e b r a$ and $F$ be a subset of $A$. Then the following conditions are equivalent:

(1) $F$ is a type-II filter of $A$;

(2) $1 \in F$ and if $a \in F$ and $a \rightsquigarrow b \in F$, then $b \in F$.

Remark 2. If $A$ is a $P Q W$-algebra, we know that the relations $\leq_{1}$ and $\leq_{2}$ coincide, so type-I filter and type-II filter are same. We call it filter for short.

We denote $\mathfrak{F}_{I}(A)\left(\mathfrak{F}_{I I}(A)\right)$ the set of all type-I filters (type-II filters) of $A$. For any subset $X \subseteq A$, the smallest type-I filter of $A$ which contains $X$, i.e., the intersection of all type-I filters $F$ such that $X \subseteq F$ is said to be the type-I filter generated by $X$ and is denoted by $[X)_{I}$. If $X$ is the singleton $\{x\}$, it follows the customary practice of writing $[x)_{I}$ instead of $[\{x\})_{I}$ and the type-I filter $[x)_{I}$ is called the principal type-I filter generated by $x$. We can give the similar definitions for type-II filters. 
Proposition 8. Let $A$ be a weak PQW-algebra. Then we have

(1) If $X$ is a type-I filter, then $[X)_{I}=X$;

(2) If $X=\{1\}$, then $[1)_{I}=\left\{a \in A \mid 1 \leq_{1} a\right\}$.

Proof. (1) It is obvious.

(2) We denote $F=\left\{a \in A \mid 1 \leq_{1} a\right\}$. Obviously, $1 \in F$. If $a, b \in F$, then $1 \leq_{1} a$ and $1 \leq_{1} b$, it turns out that $1=1 \cdot 1 \leq_{1} 1 \cdot a=a \cdot 1 \leq_{1} a \cdot b$ by Lemma 2 , so $a \cdot b \in F$. Let $a \in F$ and $b \in A$ with $a \leq_{1} b$. Then $1 \leq_{1} a \leq_{1} b$, so $b \in F$. Hence $F$ is a type-I filter of $A$. Suppose that $F^{\prime}$ is any type-I filter of $A$ with $\{1\} \subseteq F^{\prime} \subseteq F$ and $a \in F$. Then $1 \leq_{1} a$ and since $1 \in F^{\prime}$ we have $a \in F^{\prime}$, it follows that $F \subseteq F^{\prime}$. Hence $F=F^{\prime}$ and $F=[1)_{I}$.

Proposition 9. Let $A$ be a weak PQW-algebra. Then we have

(1) If $X$ is a type-II filter, then $[X)_{I I}=X$;

(2) If $X=\{1\}$, then $[1)_{I I}=\left\{a \in A \mid 1 \leq_{2} a\right\}$.

Suppose that $A$ is a weak PQW-algebra and $\left\{F_{i}\right\}_{i \in I}$ is a family of type-I filters of $A$. We define $\wedge_{i \in I} F_{i}=\cap_{i \in I} F_{i}$. Then it is easy to see that $\wedge_{i \in I} F_{i}$ is also a type-I filter of $A$. Hence $\left(\mathfrak{\mho}_{I}(A) ; \wedge\right)$ is a join-semilattice. Similarly, $\left(\widetilde{\mho}_{I I}(A) ; \wedge\right)$ is also a join-semilattice.

For any quasi-pseudo-MV algebra, we introduced weak filters as a generalization of filters in [22]. All results can be paralleled to quasi-pseudo-Wajsberg algebras, especially, to pseudo-quasi-Wajsberg algebras. Now we extend them to weak PQW-algebras.

Definition 13. Let $A$ be a weak PQW-algebra and $F$ be a subset of $A$. Then $F$ is called a left weak filter of $A$, if the following conditions are satisfied:

(LWF1) $1 \in F$;

(LWF2) If $a, b \in F$, then $a \cdot b \in F$;

(LWF3) If $a \in F$ and $b \in A$, then $a \oplus b \in F$.

$F$ is called a right weak filter of $A$, if the following conditions are satisfied:

(RWF1) $1 \in F$;

(RWF2) If $a, b \in F$, then $a \cdot b \in F$;

(RWF3) If $a \in F$ and $b \in A$, then $b \oplus a \in F$.

Moreover, if $F$ is both a left weak filter and a right weak filter of $A$, then it is called a weak filter of $A$.

Proposition 10. Let $A$ be a weak $P Q W-a l g e b r a$. Then any type-I filter is a left weak filter and any type-II filter is a right weak filter.

Proof. Let $F$ be a type-I filter of $A$ and $a \in F, b \in A$. We need to prove that $F$ satisfies (LWF3). Since $a \leq_{1} a \oplus b$ by Lemma 1, we have $a \oplus b \in F$. If $F$ is a type-II filter of $A$, the result can be proved similarly.

However, a left weak filter (or a right weak filter) may not be a type-I filter (or a type-II filter). For example, $\{1\}$ is a weak filter of $A$. However, it is not a type-I filter. Indeed, if $\{1\}$ is a type-I filter of $A$ and $1 \neq b \in A$ with $1 \leq_{1} b$, then $b=1$, this is a contradiction.

We now present an alternative characterization of left weak filters.

Definition 14. Let $A$ be a weak $P Q W$-algebra. For all $a, b \in A$, we define $a \leq_{1} b$ iff $a \vee_{1} b=b$. 
Proposition 11. Let $A$ be a weak $P Q W$-algebra. For all $a, b \in A$, the following conditions are equivalent:

(1) $a \leq_{1} b$;

(2) $a \leq_{1} b$ and $b \in R(A)$.

Proof. (1) $\Rightarrow(2)$ Assume that $a \leq_{1} b$. Then $a \vee_{1} b=b$, we have $a \vee_{1} b=(a \rightarrow b) \rightsquigarrow b=1 \rightarrow((a \rightarrow b) \rightsquigarrow$ $b)=1 \rightarrow\left(a \vee_{1} b\right)=1 \rightarrow b$, which means $a \leq_{1} b$. Meanwhile, $b=a \vee_{1} b=1 \rightarrow b$, we get $b \in R(A)$.

$(2) \Rightarrow(1)$ Assume that $a \leq_{1} b$ and $b \in R(A)$. Then $a \vee_{1} b=1 \rightarrow b=b$ and we have $a \leq_{1} b$.

Proposition 12. Let $A$ be a weak PQW-algebra and F be a left weak filter of A. Then condition (LWF3) in Definition 13 is equivalent to

(LWF3') If $a \in F$ and $b \in A$ with $a \leq_{1} b$, then $b \in F$.

Proof. Let $a \in F$ and $b \in A$ with $a \leq_{1} b$. Then $b=a \vee_{1} b=a \oplus\left(a^{\prime} \cdot b\right) \in F$ by (LWF2). Conversely, let $a \in F$ and $b \in A$, we have $a \leq_{1} a \oplus b$ by Lemma 1 and $a \oplus b \in R(A)$. Then $a \leq_{1} a \oplus b$ by Proposition 11, it turns out that $a \oplus b \in F$.

Similarly, we present an alternative characterization of right weak filters.

Definition 15. Let $A$ be a weak $P Q W$-algebra. For all $a, b \in A$, we define $a \leq_{2} b$ iff $a \vee_{2} b=b$.

Proposition 13. Let $A$ be a weak $P Q W$-algebra. For all $a, b \in A$, the following conditions are equivalent:

(1) $a \leq_{2} b$;

(2) $a \leq_{2} b$ and $b \in R(A)$.

Proposition 14. Let $A$ be a weak PQW-algebra and $F$ be a right weak filter of $A$. Then condition (RWF3) in Definition 13 is equivalent to

(RWF3') If $a \in F$ and $b \in A$ with $a \leq_{2} b$, then $b \in F$.

Definition 16. Let $A$ and $B$ be weak $P Q W$-algebras. A mapping $f: A \rightarrow B$ is called a weak PQW-algebra homomorphism if the following conditions are satisfied for all $a, b \in A$ :

(H1) $f(a \rightarrow b)=f(a) \rightarrow f(b)$;

(H2) $f(a \rightsquigarrow b)=f(a) \rightsquigarrow f(b)$;

(H3) $f\left(a^{\prime}\right)=(f(a))^{\prime}$;

(H4) $f(1)=1$.

Remark 3. If $f$ is a weak $P Q W$-algebra homomorphism from $A$ to $B$, then $f(0)=0$. Indeed, we have $f(0)=$ $f\left(1^{\prime}\right)=(f(1))^{\prime}=1^{\prime}=0$ by $(H 3)$ and $(H 4)$.

Proposition 15. Let $A$ and $B$ be weak $P Q W-a l g e b r a s$ and $f$ be a weak $P Q W$-algebra homomorphism from $A$ to B. Then

(1) $f(a \oplus b)=f(a) \oplus f(b)$;

(2) $f(a \cdot b)=f(a) \cdot f(b)$;

(3) $a \leq_{1} b \Rightarrow f(a) \leq_{1} f(b)$;

(4) $a \leq_{2} b \Rightarrow f(a) \leq_{2} f(b)$;

(5) $f\left(a \vee_{1} b\right)=f(a) \vee_{1} f(b)$; 
(6) $f\left(a \vee_{2} b\right)=f(a) \vee_{2} f(y)$;

(7) $f\left(a \wedge_{1} b\right)=f(a) \wedge_{1} f(b)$;

(8) $f\left(a \wedge_{2} b\right)=f(a) \wedge_{2} f(b)$.

Proof. (1) We have $f(a \oplus b)=f\left(a^{\prime} \rightarrow b\right)=f\left(a^{\prime}\right) \rightarrow f(b)=(f(a))^{\prime} \rightarrow f(b)=f(a) \oplus f(b)$ by (H1) and (H3).

(2) We have $f(a \cdot b)=f\left(\left(b^{\prime} \oplus a^{\prime}\right)^{\prime}\right)=\left(f(b)^{\prime} \oplus f(a)^{\prime}\right)^{\prime}=f(a) \cdot f(b)$ by (1) and (H3).

(3) Since $a \leq_{1} b$, we have $f(a) \rightarrow f(b)=f(a \rightarrow b)=f(1)=1$ by (H1) and (H4), it follows that $f(a) \leq_{1} f(b)$.

(4) Since $a \leq_{2} b$, we have $f(a) \rightsquigarrow f(b)=f(a \rightsquigarrow b)=f(1)=1$ by (H2) and (H4), it follows that $f(a) \leq_{2} f(b)$.

(5) We have $f\left(a \vee_{1} b\right)=f((a \rightarrow b) \rightsquigarrow b)=f(a \rightarrow b) \rightsquigarrow f(a)=(f(a) \rightarrow f(b)) \rightsquigarrow f(b)=f(a) \vee_{1} f(b)$ by (H1) and (H2).

(6) It is similar to (5).

(7) We have $f\left(a \wedge_{1} b\right)=f\left(\left(a^{\prime} \vee_{2} b^{\prime}\right)^{\prime}\right)=\left(f\left(a^{\prime} \vee_{2} b^{\prime}\right)\right)^{\prime}=\left(f\left(a^{\prime}\right) \vee_{2} f\left(b^{\prime}\right)\right)^{\prime}=\left((f(a))^{\prime} \vee_{2}(f(b))^{\prime}\right)^{\prime}=$ $f(a) \wedge_{1} f(b)$ by $(\mathrm{H} 3)$ and (6).

(8) It is similar to (7).

Proposition 16. Let $A$ and $B$ be weak $P Q W$-algebras and $f$ be a weak $P Q W$-algebra homomorphism from $A$ to $B$. Then

(1) If $F$ is a type-I filter of $B$, then $f^{-1}(F)$ is a type-I filter of $A$.

(2) If $F$ is a type-II filter of $B$, then $f^{-1}(F)$ is a type-II filter of $A$.

(3) If $F$ is a left weak filter of $B$, then $f^{-1}(F)$ is a left weak filter of $A$.

(4) If $F$ is a right weak filter of $B$, then $f^{-1}(F)$ is a right weak filter of $A$.

Proof. (1) Obviously, $1 \in f^{-1}(F)$ and $f^{-1}(F)$ is non-empty subset of $A$. For all $a, b \in f^{-1}(F)$, we have $f(a), f(b) \in F$, it follows that $f(a \cdot b)=f(a) \cdot f(b) \in F$ by Proposition 15 (2). Thus $a \cdot b \in f^{-1}(F)$. Let $a \in f^{-1}(F)$ and $y \in A$ with $a \leq_{1} b$. Then $f(a) \in F$ and $f(a) \leq_{1} f(b)$, it follows that $f(b) \in F$. Thus $b \in f^{-1}(F)$.

(2) It is similar to (1).

(3) We only show that $f^{-1}(F)$ satisfies (LWF3). For all $a \in f^{-1}(F)$ and $b \in A$, we have $f(a) \in F$ and then $f(a \oplus b)=f(a) \oplus f(b) \in F$. Thus $a \oplus b \in f^{-1}(F)$.

(4) It is dual to (3).

Proposition 17. Let $A$ and $B$ be weak $P Q W$-algebras and $f$ be a weak $P Q W$-algebra epimorphism from $A$ to $B$. Then

(1) If $F$ is a left weak filter of $A$, then $f(F)$ is a left weak filter of $B$.

(2) If $F$ is a right weak filter of $A$, then $f(F)$ is a right weak filter of $B$.

(3) If $F$ is a weak filter of $A$, then $f(F)$ is a weak filter of $B$.

Proof. (1) Obviously, $1=f(1) \in f(F)$. For all $a, b \in f(F)$, then there exist $m, n \in F$ such that $f(m)=a$ and $f(n)=b$. Since $F$ is a left weak filter of $A$, we have $m \cdot n \in F$. Thus $a \cdot b=f(m) \cdot f(n)=f(m \cdot n) \in f(F)$ by Proposition 15 (2). Let $a \in f(F)$ and $b \in B$. Then there exist $m \in F$ and $n \in A$ such that $f(m)=a$ and $f(n)=b$. Since $F$ is a left weak filter of $A$, we have $m \oplus n \in F$, thus $a \oplus b=f(m) \oplus f(n)=f(m \oplus n) \in f(F)$ by Proposition $15(1)$. Hence $f(F)$ is a left weak filter of $A$.

(2) It is similar to (1).

(3) By (1) and (2). 
Definition 17. Let $A$ be a weak $P Q W$-algebra and $F$ be a left weak filter or right weak filter of $A$. Then $F$ is called normal, if for all $a, b \in A$, we have $a \rightarrow b \in F \Leftrightarrow a \rightsquigarrow b \in F$.

Remark 4. Any normal left weak filter is a weak filter. Indeed, let $F$ be a normal left weak filter of $A$. If $a \in F$ and $b \in A$, then $a \oplus b=a^{\prime} \rightarrow b \in F$. Since $F$ is normal, we have $a^{\prime} \rightsquigarrow b \in F$, it follows that $b \oplus a=b^{\prime} \rightarrow a=a^{\prime} \rightsquigarrow b \in F$, which means that $F$ is a right weak filter of $A$. Hence $F$ is a weak filter of $A$. Similarly, any normal right weak filter is a weak filter.

Remark 5. Any normal type-I filter is a type-II filter. Indeed, let $F$ be a normal type-I filter of $A$. If $a \in F$ and $a \rightsquigarrow b \in F$, then $a \rightarrow b \in F$, it follows that $b \in F$. So F is a type-II filter of A. Similarly, any normal type-II filter is type-I filter. Hence we call it normal filter for short.

Definition 18. Let $A$ be a weak $P Q W$-algebra and $\theta$ be a congruence on $A$. Then $\theta$ is called a filter congruence, if for all $a, b \in A,\langle 1 \rightarrow a, 1 \rightarrow b\rangle \in \theta$ can imply $\langle a, b\rangle \in \theta$.

Proposition 18. Let $A$ be a weak $P Q W$-algebra and $\theta$ be a filter congruence on $A$. Then $F_{\theta}=\{a \in A \mid\langle a, 1\rangle \in \theta\}$ is a type-I filter and type-II filter of $A$, so is a weak filter of $A$.

Proof. Obviously, $1 \in F_{\theta}$. If $a, b \in F_{\theta}$, then $\langle a, 1\rangle \in \theta$ and $\langle b, 1\rangle \in \theta$, it follows that $\langle a \cdot b, 1 \cdot 1\rangle=\langle a \cdot b, 1\rangle \in \theta$, so $a \cdot b \in F_{\theta}$. Let $a \in F_{\theta}$ and $b \in A$ with $a \leq_{1} b$. Then $\langle a, 1\rangle \in \theta$ and then $\langle(a \rightarrow b) \rightsquigarrow b,(1 \rightarrow b) \rightsquigarrow b\rangle \in \theta$. Because $a \vee_{1} b=(a \rightarrow b) \rightsquigarrow b=1 \rightarrow b$ and $1 \vee_{1} b=(1 \rightarrow b) \rightsquigarrow b=1 \rightarrow 1$, we have $\langle 1 \rightarrow b, 1 \rightarrow 1\rangle \in \theta$. Please note that $\theta$ is a filter congruence on $A$, so $\langle b, 1\rangle \in \theta$ and $b \in F_{\theta}$. Hence $F_{\theta}$ is a type-I filter of $A$. Similarly, if $a \in F_{\theta}$ and $b \in A$ with $a \leq_{2} b$, then $b \in F_{\theta}$. Hence $F_{\theta}$ is a type-II filter of $A$. By Proposition 10, it is a weak filter of $A$.

Remark 6. Let $A$ be a weak $P Q W$-algebra. If $\theta$ is only a congruence on $A$, then $F_{\theta}=\{a \in A \mid\langle a, 1\rangle \in \theta\}$ may not be a type-I or type-II filter of $A$, but must be a weak filter of $A$. Because if $a \in F_{\theta}$ and $b \in A$, then $\langle a, 1\rangle \in \theta$ and $\langle a \oplus b, 1 \oplus b\rangle=\langle a \oplus b, 1\rangle \in \theta$, so $a \oplus b \in F_{\theta}$. Similarly, we have $\langle b \oplus a, b \oplus 1\rangle=\langle b \oplus a, 1\rangle \in \theta$, so $b \oplus a \in F_{\theta}$. Hence $F_{\theta}$ is a weak filter of $A$.

In [22], we had proved that if $A$ is a PQW-algebra and $\theta$ is a filter congruence on $A$, then $F_{\theta}$ is a normal filter of $A$. In addition, if $F$ is a normal filter of $A$ and we define $\theta_{F}=\{\langle a, b\rangle \mid a \rightarrow b \in F$ and $b \rightarrow a \in F\}$, then $\theta_{F}$ is a filter congruence on $A$. However, it is hard for us to show the similar result for a weak PQW-algebra. Below we strengthen the condition of normal filters.

Definition 19. Let $A$ be a weak $P Q W$-algebra and $F$ be a type-I filter (or type-II filter) of $A$. Then $F$ is called strong normal, if for all $a, b, c \in A$, we have $a \rightarrow(b \rightsquigarrow c) \in F$ iff $a \rightarrow(b \rightarrow c) \in F$ and $a \rightsquigarrow(b \rightarrow c) \in F$ iff $a \rightsquigarrow(b \rightsquigarrow c) \in F$.

Proposition 19. Any strong normal type-I filter is normal.

Proof. For any $a, b \in A$ and if $a \rightarrow b \in F$, then $1 \rightarrow(a \rightarrow b)=a \rightarrow b \in F$. Since $F$ is strong normal, we have $1 \rightarrow(a \rightsquigarrow b) \in F$ and then $a \rightsquigarrow b=1 \rightsquigarrow(a \rightsquigarrow b)=1 \rightarrow(a \rightsquigarrow b) \in F$. The converse can be proved similarly.

Proposition 20. Let $A$ be a weak $P Q W$-algebra and $F$ be a strong normal type-I filter of $A$. Define $\theta_{F}=\{\langle a, b\rangle \in$ $A^{2} \mid a \rightarrow b \in$ Fand $\left.b \rightarrow a \in F\right\}$. Then $\theta_{F}$ is a filter congruence on $A$. 
Proof. Obviously, the relation $\theta_{F}$ is reflexivity, symmetry and filter. For all $\langle a, b\rangle \in \theta_{F}$ and $\langle b, c\rangle \in \theta_{F}$, then $((b \rightarrow c) \odot(a \rightarrow b)) \rightarrow(a \rightarrow c)=\left((a \rightarrow b) \rightarrow(b \rightarrow c)^{\prime}\right)^{\prime} \rightarrow(a \rightarrow c)=(a \rightarrow c)^{\prime} \rightsquigarrow((a \rightarrow$ $\left.b) \rightarrow(b \rightarrow c)^{\prime}\right)=(a \rightarrow b) \rightarrow\left((a \rightarrow c)^{\prime} \rightsquigarrow(b \rightarrow c)^{\prime}\right)=(a \rightarrow b) \rightarrow((b \rightarrow c) \rightarrow(a \rightarrow c))$. Since $(a \rightarrow b) \rightarrow((b \rightarrow c) \rightsquigarrow(a \rightarrow c))=1 \in F$ and $F$ is strong normal, we have $((b \rightarrow c) \odot(a \rightarrow b)) \rightarrow(a \rightarrow$ $c) \in F$. Because $(b \rightarrow c) \odot(a \rightarrow b) \in F$, it follows that $a \rightarrow c \in F$ by Proposition 6. Similarly, we have $((c \rightsquigarrow b) \odot(b \rightsquigarrow a)) \rightsquigarrow(c \rightsquigarrow a) \in F$ and then $c \rightsquigarrow a \in F$. By Proposition 19, $c \rightarrow a \in F$. Hence $\theta_{F}$ is an equivalence on $A$. Let $\langle a, b\rangle \in \theta_{F}$ and all $a \in A$. Then by (P23), we have $a \rightarrow b \leq_{1}(b \rightarrow a) \rightsquigarrow(a \rightarrow a)$ and $(b \rightarrow a) \rightsquigarrow(a \rightarrow a) \in F$. Using Proposition 19, we have $(b \rightarrow a) \rightarrow(a \rightarrow a) \in F$. Similarly, we can show that $(a \rightarrow a) \rightarrow(b \rightarrow a) \in F$, so $\langle a \rightarrow a, b \rightarrow a\rangle \in \theta_{F}$. Dually, we have $\langle a \rightsquigarrow a, b \rightsquigarrow a\rangle \in \theta_{F}$. On the other hand, we can prove $\langle a \rightarrow a, a \rightarrow b\rangle \in \theta_{F}$ and $\langle a \rightsquigarrow a, a \rightsquigarrow b\rangle$ using (P24). Finally, since $a^{\prime} \rightarrow b^{\prime}=b \rightsquigarrow a$ and $F$ is strong normal, we have $\left\langle a^{\prime}, b^{\prime}\right\rangle \in \theta_{F}$. Thus $\theta_{F}$ is a filter congruence on $A$.

Let $A$ be a weak PQW-algebra and $\theta$ be a congruence on $A$. The set $A / \theta=\{a / \theta \mid a \in A\}$, where $a / \theta=\{b \in A \mid\langle a, b\rangle \in \theta\}$ is called the quotient set of $A$ with respect to $\theta$. We define some operations on $A / \theta$ as follows:

(1) $a / \theta \rightarrow b / \theta=(a \rightarrow b) / \theta$;

(2) $a / \theta \rightsquigarrow b / \theta=(a \rightsquigarrow b) / \theta$;

(3) $(a / \theta)^{\prime}=a^{\prime} / \theta$.

Then it is easy to see that the algebraic structure of $A / \theta$ is inherited from the algebra $A$. So it is a weak PQW-algebra.

Lemma 3. Let $A$ be a weak $P Q W$-algebra and $\theta$ be a filter congruence on $A$. Then $1 / \theta \rightarrow a / \theta=a / \theta=1 / \theta \leadsto$ $a / \theta$.

Proof. By (P18), we have $\langle 1 \rightarrow(1 \rightarrow a), 1 \rightarrow a\rangle=\langle 1 \rightarrow a, 1 \rightarrow a\rangle \in \theta$. Since $\theta$ is a filter congruence on $A$, we get $\langle 1 \rightarrow a, a\rangle \in \theta$, so $1 / \theta \rightarrow a / \theta=(1 \rightarrow a) / \theta=a / \theta$. The other can be proved similarly.

Proposition 21. Let $A$ be a weak $P Q W$-algebra and $\theta$ be a filter congruence on $A$. Then $A / \theta=\left\{A / \theta ; \rightarrow, \rightsquigarrow,{ }^{\prime}, 1 / \theta\right\}$ is a weak pseudo-Wajsberg algebra.

Proof. Follows from $A / \theta$ is a weak PQW-algebra and Lemma 3.

Let $A$ and $B$ be weak PQW-algebras and $f$ be a weak PQW-algebra homomorphism from $A$ to $B$. We denote $\operatorname{ker}(f)=\left\{\langle a, b\rangle \in A^{2} \mid f(a)=f(b)\right\}$. Then $\operatorname{ker}(f)$ is a congruence on $A$ by Theorem 6.8 in [21].

Proposition 22. Let $A$ be a weak $P Q W$-algebra and $\theta$ be a filter congruence on $A$. Then the mapping $\varphi: A \rightarrow A / \theta$ is a surjective homomorphism and $\operatorname{ker}(\varphi)=\theta$ is a filter congruence on $A$.

Proof. Obviously, $\varphi$ is surjective and $\varphi$ satisfies

(H1) $\varphi(a \rightarrow b)=(a \rightarrow b) / \theta=a / \theta \rightarrow b / \theta=\varphi(a) \rightarrow \varphi(b)$.

(H2) $\varphi(a \rightsquigarrow b)=(a \rightsquigarrow b) / \theta=a / \theta \rightsquigarrow b / \theta=\varphi(a) \rightsquigarrow \varphi(b)$.

(H3) $\varphi\left(a^{\prime}\right)=a^{\prime} / \theta=(a / \theta)^{\prime}=(\varphi(a))^{\prime}$.

(H4) $\varphi(1)=1 / \theta$.

Next, we show that $\operatorname{ker}(\varphi)$ is a filter congruence on $A$. Indeed, for all $\langle 1 \rightarrow a, 1 \rightarrow b\rangle \in \operatorname{ker}(\varphi)$, then $1 \rightarrow \varphi(a)=1 \rightarrow \varphi(b)$. Since $A$ / $\theta$ is a weak pseudo-Wajsberg algebra by Proposition 21, we have 
$\varphi(a)=\varphi(b)$, so $\langle a, b\rangle \in \operatorname{ker}(\varphi)$. Finally, for all $\langle a, b\rangle \in \operatorname{ker}(\varphi)$ iff $\varphi(a)=\varphi(b)$ iff $a / \theta=b / \theta$ iff $\langle a, b\rangle \in \theta$. Hence $\operatorname{ker}(\varphi)=\theta$.

\section{Weak Pseudo-Quasi-Wajsberg Algebras and Residuated Quasi-Ordered Monoid Structures}

In this section, the concepts of some residuated quasi-ordered monoid structures are introduced and the relationship between weak PQW-algebras and them is investigated.

Definition 20. Let $A=\langle A ; \cdot, \leq, 1\rangle$ be an algebra of type $\langle 2,2,0\rangle$. Then it is called a left partial ordered monoid, if the following conditions are satisfied for all $a, b, c \in A$ :

(1) (monoid) (1.1) $(a \cdot b) \cdot c=a \cdot(b \cdot c),(1.2) a \cdot 1=a=1 \cdot a$;

(2) (partial ordered) (2.1) $a \leq a$ (reflexivity),

(2.2) If $a \leq b$ and $b \leq a$, then $a=b$ (antisymmetry),

(2.3) If $a \leq b$ and $b \leq c$, then $a \leq c$ (transitivity);

(3) If $a \leq b$, then $x \cdot a \leq x \cdot b$ for all $x \in A$.

Similarly, we can define right partial ordered monoids.

Definition 21. Let $A=\langle A ; \cdot \leq, 1\rangle$ be an algebra of type $\langle 2,2,0\rangle$. Then it is called a right partial ordered monoid, if the following conditions are satisfied for all $a, b, c \in A$ :

(1) (monoid) (1.1) $(a \cdot b) \cdot c=a \cdot(b \cdot c),(1.2) a \cdot 1=a=1 \cdot a$;

(2) (partial ordered) (2.1) $a \leq a$ (reflexivity),

(2.2) If $a \leq b$ and $b \leq a$, then $a=b$ (antisymmetry),

(2.3) If $a \leq b$ and $b \leq c$, then $a \leq c$ (transitivity);

(3) If $a \leq b$, then $a \cdot x \leq b \cdot x$ for all $x \in A$.

Remark 7. If $\langle A ; \cdot\rangle$ is an abelian monoid in Definition 20 or Definition 21, then left and right partial ordered monoid are same, that is, a partial ordered abelian monoid. More generally, if $A$ is both a left partial ordered monoid and a right partial ordered monoid, then it is a partial ordered monoid.

Below we give the definitions of left quasi-ordered monoid and right quasi-ordered monoid which generalize left partial ordered monoid and right partial ordered monoid, respectively.

Definition 22. Let $A=\langle A ; \cdot, \leq, 1\rangle$ be an algebra of type $\langle 2,2,0\rangle$. Then it is called a left quasi-ordered quasi-monoid (left quasi-ordered monoid, for short), if the following conditions are satisfied for all $a, b, c \in A$ :

(1) (quasi-monoid) (1.1) $(a \cdot b) \cdot c=a \cdot(b \cdot c),(1.2) a \cdot 1=1 \cdot a$,

(1.3) $1 \cdot(a \cdot b)=a \cdot b,(1.4) 1 \cdot 1=1$;

(2) (quasi-ordered) (2.1) $a \leq a$ (reflexivity),

(2.2) If $a \leq b$ and $b \leq a$, then $1 \cdot a=1 \cdot b$ (quasi-antisymmetry),

(2.3) If $a \leq b$ and $b \leq c$, then $a \leq c$ (transitivity);

(3) If $a \leq b$, then $x \cdot a \leq x \cdot b$ for all $x \in A$;

(4) $a \leq 1 \cdot a$ and $1 \cdot a \leq a$.

Dually, we have the following definition.

Definition 23. Let $A=\langle A ; \cdot, \leq, 1\rangle$ be an algebra of type $\langle 2,2,0\rangle$. Then it is called a right quasi-ordered quasi-monoid (right quasi-ordered monoid, for short), if it satisfies the following conditions for all $a, b, c \in A$ : 
(1) (quasi-monoid) (1.1) $(a \cdot b) \cdot c=a \cdot(b \cdot c)$, (1.2) $a \cdot 1=1 \cdot a$, (1.3) $(a \cdot b) \cdot 1=a \cdot b,(1.4) 1 \cdot 1=1$;

(2) (quasi-ordered) (2.1) $a \leq a$ (reflexivity),

(2.2) If $a \leq b$ and $b \leq a$, then $a \cdot 1=b \cdot 1$ (quasi-antisymmetry),

(2.3) If $a \leq b$ and $b \leq c$, then $a \leq c$ (transitivity);

(3) If $a \leq b$, then $a \cdot x \leq b \cdot x$ for all $x \in A$;

(4) $a \leq a \cdot 1$ and $a \cdot 1 \leq a$.

Remark 8. If $\langle A ; \cdot\rangle$ is an abelian quasi-monoid in Definition 22 or Definition 23, then left quasi-ordered monoid and right quasi-ordered monoid are same. If $A$ is both a left quasi-ordered monoid and a right quasi-ordered monoid, then it is a quasi-ordered monoid defined in [24].

Remark 9. Let $A$ be a left (right) quasi-ordered monoid. If $\langle A ; \cdot, 1\rangle$ is a monoid, then $a \cdot 1=a$, which implies that $\langle A ; \leq, 1\rangle$ is a partial ordering, so $A$ is a left (right) partial ordered monoid. If $\langle A ; \leq, 1\rangle$ is a partial ordering, then by the condition (4), we get that $a \cdot 1=a$, which implies that $\langle A ; \cdot, 1\rangle$ is a monoid, so $A$ is also a left (right) partial ordered monoid.

Let $A$ be a left quasi-ordered monoid. We denote the set $1 \cdot A=\{a \in A \mid 1 \cdot a=a\}$. Obviously, $1 \in 1 \cdot A$ and the set is non-empty. Based on Remark 9, we have that $1 \cdot A$ is a left partial ordered monoid. Similarly, let $A$ be a right quasi-ordered monoid. We denote the set $A \cdot 1=\{a \in A \mid a \cdot 1=a\}$. Then it is a right partial ordered monoid. The elements in $1 \cdot A$ and $A \cdot 1$ are called regular. In addition, if a left (right) quasi-ordered monoid is with the lower bound 0 and the upper bound 1 , then we call it bounded.

Now we discuss the relationship between left (right) quasi-ordered monoids and weak PQW-algebras.

Definition 24. Let $A=\langle A ; \cdot, \leq, 1\rangle$ be a left quasi-ordered monoid. Then $A$ is called right residuated if for all $a, b \in A$, there is a greatest regular element $x$ with $a \cdot x \leq b$. We will denote this element by $a \rightarrow b$. A is called left residuated if for all $a, b \in A$, there is a greatest regular element $y$ with $y \cdot a \leq b$. We will denote this element by $a \rightsquigarrow b$. Similarly, if $A=\langle A ; \cdot, \leq, 1\rangle$ is a right quasi-ordered monoid, we can also define right residuated and left residuated as above.

Proposition 23. Let $A=\langle A ; \rightarrow, n \rightarrow,, 1\rangle$ be a weak PQW-algebra. If $a \leq_{1} b$ is defined by $a \rightarrow b=1, a \leq 2 b$ is defined by $a \rightsquigarrow b=1$ and $0=1^{\prime}$, then

(1) $\left\langle A ; \cdot, \leq_{1}, 0,1\right\rangle$ is a bounded left quasi-ordered monoid.

(2) $\left\langle A ; \cdot, \leq_{2}, 0,1\right\rangle$ is a bounded right quasi-ordered monoid.

Proof. (1) We have known that $\leq_{1}$ is reflexive and transitive [19]. Here we only show the quasi-antisymmetry. If $a \leq_{1} b$ and $b \leq_{1} a$, then $b^{\prime} \leq_{2} a^{\prime}$ and $a^{\prime} \leq_{2} b^{\prime}$ by (P29), it follows that $1 \rightsquigarrow a^{\prime}=1 \rightsquigarrow b^{\prime}$ by (P1). Thus $1 \cdot a=\left(1 \rightsquigarrow a^{\prime}\right)^{\prime}=\left(1 \rightsquigarrow b^{\prime}\right)^{\prime}=1 \cdot b$. Moreover, by Lemma 2 , the operation $\cdot$ is monotony.

It still needs to prove that $\langle A ; \cdot, 1\rangle$ is quasi-monoid. For $(1.1)$, we have $a \cdot 1=\left(1 \rightarrow a^{\prime}\right)^{\prime}=(1 \mathrm{~m}$ $\left.a^{\prime}\right)^{\prime}=1 \cdot a$ by (wPQW1). For (1.2), we have $(a \cdot b) \cdot c=\left[c \rightarrow(a \cdot b)^{\prime}\right]^{\prime}=\left[c \rightarrow\left(a \rightsquigarrow b^{\prime}\right)\right]^{\prime}=[a \rightsquigarrow(c \rightarrow$ $\left.\left.b^{\prime}\right)\right]^{\prime}=a \cdot\left(c \rightarrow b^{\prime}\right)^{\prime}=a \cdot(b \cdot c)$ by (wPQW9). For (1.3), we have $(a \cdot b) \cdot 1=\left[1 \rightsquigarrow(a \cdot b)^{\prime}\right]^{\prime}=[1 \rightsquigarrow(b \rightarrow$ $\left.\left.a^{\prime}\right)\right]^{\prime}=\left(b \rightarrow a^{\prime}\right)^{\prime}=a \cdot b$ by (wPQW2). For (1.4), we have $1 \cdot 1=\left(1 \rightarrow 1^{\prime}\right)^{\prime}=1^{\prime \prime}=1$ by (P20) and (wPQW8). For (4), we have $(1 \cdot a) \rightarrow a=\left(a \rightarrow 1^{\prime}\right)^{\prime} \rightarrow a=a^{\prime} \rightsquigarrow\left(1 \rightsquigarrow a^{\prime}\right)=1$ by (wPQW6) and (P16) and $a \rightarrow(1 \cdot a)=a \rightarrow\left(a \rightarrow 1^{\prime}\right)^{\prime}=\left(1 \rightsquigarrow a^{\prime}\right) \rightsquigarrow a^{\prime}=1$ by (wPQW6), (wPQW1) and (P6), it turns that $1 \cdot a \leq{ }_{1} a$ and $a \leq_{1} 1 \cdot a$. 
Finally, we have $a \rightarrow 1=1$ by (P15), it follows that $a \leq_{1} 1$. Meanwhile, put $0=1^{\prime}$, then $0 \rightarrow a=1^{\prime} \rightarrow a=a^{\prime} \rightsquigarrow 1=1$ by (wPQW6) and (P15), it follows that $0 \leq_{1} a$. Hence $A$ is bounded.

(2) It is similar to (1).

Proposition 24. Let $A=\left\langle A ; \rightarrow, \rightsquigarrow{ }^{\prime}, 1\right\rangle$ be a weak $P Q W$-algebra. If $a \leq_{1} b$ is defined by $a \rightarrow b=1$ and $a \leq_{2} b$ is defined by $a \rightsquigarrow b=1$, then we have

(1) The operation $\rightarrow$ is the right residual of $\cdot$, i.e., we have $a \cdot b \leq_{1} c$ iff $b \leq_{1} a \rightarrow c$;

(2) The operation $\rightsquigarrow$ is the left residual of $\cdot$, i.e., we have $a \cdot b \leq_{2} c$ iff $a \leq_{2} b \leadsto c$.

Proof. (1) We calculate $(a \cdot b) \rightarrow c=\left(b \rightarrow a^{\prime}\right)^{\prime} \rightarrow c=c^{\prime} \rightsquigarrow\left(b \rightarrow a^{\prime}\right)=b \rightarrow\left(c^{\prime} \rightsquigarrow a^{\prime}\right)=b \rightarrow(a \rightarrow c)$. From this, we have the desired equivalence.

(2) It can be proved similarly as (1).

Theorem 3. Let $A=\left\langle A ; \rightarrow, \rightsquigarrow,^{\prime}, 1\right\rangle$ be a weak PQW-algebra. If $a \leq_{1} b$ is defined by $a \rightarrow b=1, a \leq_{2} b$ is defined by $a \rightsquigarrow b=1$ and $0=1^{\prime}$, then

(1) $\left\langle A ; \cdot, \leq_{1}, 0,1\right\rangle$ is a bounded left quasi-ordered monoid with right residual;

(2) $\left\langle A ; \cdot, \leq_{2}, 0,1\right\rangle$ is a bounded right quasi-ordered monoid with left residual.

Proof. Follows from Propositions 23 and 24.

Now it is natural to ask under which conditions left quasi-ordered monoid or right quasi-ordered monoid becomes a weak PQW-algebra. To do these, we need to discuss some properties of left quasi-ordered monoid and right quasi-ordered monoid.

Proposition 25. Let $A=\langle A ; \cdot, \leq, 0,1\rangle$ be a bounded left quasi-ordered monoid. If $A$ is right residuated, then

(1) It has $\rightarrow$ as the right residual of $\cdot$, which satisfies $a \cdot b \leq c \Leftrightarrow b \leq a \rightarrow c$ and $a \cdot(a \rightarrow b) \leq b$;

(2) $a \leq b \Leftrightarrow a \rightarrow b=1$;

(3) If $a \leq b$, then $c \rightarrow a \leq c \rightarrow b$;

(4) If $a \leq b$ and $b \leq a$, then $1 \rightarrow a=1 \rightarrow b$;

(5) $a \rightarrow b=(1 \cdot a) \rightarrow b=(a \cdot 1) \rightarrow b=a \rightarrow(1 \cdot b)=a \rightarrow(b \cdot 1)$.

If $A$ is left residuated, then

(6) It has $\rightsquigarrow$ as the left residual of $\cdot$, which satisfies $a \cdot b \leq c \Rightarrow a \leq b \rightsquigarrow c$ and $(b \rightsquigarrow c) \cdot b \leq c$;

(7) $a \leq b \Leftrightarrow a \rightsquigarrow b=1$;

(8) If $a \leq b$, then $b \rightsquigarrow c \leq a \rightsquigarrow c$ and $c \rightsquigarrow a \leq c \rightsquigarrow b$;

(9) If $a \leq b$ and $b \leq a$, then $1 \rightsquigarrow a=1 \leadsto b$;

(10) $a \rightsquigarrow b=(a \cdot 1) \rightsquigarrow b=(1 \cdot a) \rightsquigarrow b=a \rightsquigarrow(1 \cdot b)=a \rightsquigarrow(b \cdot 1)$.

Proof. (1) If $a \cdot b \leq c$, then there is a greatest regular element $a \rightarrow c$ with $a \cdot(a \rightarrow c) \leq c$. Please note that $a \cdot b=a \cdot b \cdot 1 \leq c$ and $b \cdot 1$ is a regular element, we have $b \cdot 1 \leq a \rightarrow c$. Since $b \leq b \cdot 1$, we have $b \leq a \rightarrow c$. Conversely, if $b \leq a \rightarrow c$, then $a \cdot b \leq a \cdot(a \rightarrow c) \leq c$.

(2) If $a \leq b$, then $a \cdot 1 \leq b$, it turns out that $1 \leq a \rightarrow b$. In addition, $a \rightarrow b \leq 1$, we have $1 \cdot 1=1 \cdot(a \rightarrow b)$ by the quasi-antisymmetry. Please note that 1 and $a \rightarrow b$ are regular elements, we have $1=a \rightarrow b$. Conversely, if $a \rightarrow b=1$, since $a \cdot(a \rightarrow b) \leq b$ by (1), we have $a \cdot 1 \leq b$. In addition, $a \leq a \cdot 1$, we get $a \leq b$ by the transitivity. 
(3) If $a \leq b$, since $c \cdot(c \rightarrow a) \leq a$ by (1), we have $c \cdot(c \rightarrow a) \leq b$ by the transitivity, it follows that $c \rightarrow a \leq c \rightarrow b$.

(4) If $a \leq b$ and $b \leq a$, then $1 \rightarrow a \leq 1 \rightarrow b$ and $1 \rightarrow b \leq 1 \rightarrow a$ by (3), we have $1 \cdot(1 \rightarrow a)=1 \cdot(1 \rightarrow b)$. Please note that $1 \rightarrow a$ and $1 \rightarrow b$ are regular elements, so $1 \rightarrow a=1 \rightarrow b$.

(5) By (1), we have $(1 \cdot a) \cdot((1 \cdot a) \rightarrow b) \leq b$. Because $(1 \cdot a) \cdot((1 \cdot a) \rightarrow b)=a \cdot((1 \cdot a) \rightarrow b)$, we have $(1 \cdot a) \rightarrow b \leq a \rightarrow b$. Similarly, $(1 \cdot a) \cdot(a \rightarrow b)=a \cdot(a \rightarrow b) \leq b$, it follows that $a \rightarrow b \leq(1 \cdot a) \rightarrow b$. Hence $1 \cdot((1 \cdot a) \rightarrow b)=1 \cdot(a \rightarrow b)$ and then $(1 \cdot a) \rightarrow b=a \rightarrow b$. Please note that $1 \cdot a=a \cdot 1$, we have $a \rightarrow b=(a \cdot 1) \rightarrow b$. On the other hand, since $b \leq 1 \cdot b$ and $1 \cdot b \leq b$, we have $a \rightarrow b \leq a \rightarrow(1 \cdot b)$ and $a \rightarrow(1 \cdot b) \leq a \rightarrow b$, it follows that $1 \cdot(a \rightarrow b)=1 \cdot(a \rightarrow(1 \cdot b))$. Thus $a \rightarrow b=a \rightarrow(1 \cdot b)$. Please note that $1 \cdot b=b \cdot 1$, we have $a \rightarrow b=a \rightarrow(b \cdot 1)$.

(6) By the definition of left residual.

(7) If $a \leq b$, then $1 \cdot a \leq b$, it follows that $1 \leq a \rightsquigarrow b$. In addition, $a \rightsquigarrow b \leq 1$, we have $a \rightsquigarrow b=(a \rightsquigarrow$ b) $\cdot 1=1 \cdot 1=1$. Conversely, if $a \rightsquigarrow b=1$, then $a \leq 1 \cdot a=(a \rightsquigarrow b) \cdot a \leq b$.

(8) If $a \leq b$, then $(b \rightsquigarrow c) \cdot a \leq(b \rightsquigarrow c) \cdot b \leq c$, it follows that $b \rightsquigarrow c \leq a \rightsquigarrow c$. Moreover, because $(c \rightsquigarrow a) \cdot c \leq a \leq b$, we have $c \rightsquigarrow a \leq c \rightsquigarrow b$.

(9) Follows from (8).

(10) Since $a \leq a \cdot 1$ and $a \cdot 1 \leq a$, we have $(a \cdot 1) \rightsquigarrow b \leq a \rightsquigarrow b$ and $a \rightsquigarrow b \leq(a \cdot 1) \rightsquigarrow b$ by (8), it follows that $(a \rightsquigarrow b) \cdot 1=((a \cdot 1) \rightsquigarrow b) \cdot 1$. Please note that $a \rightsquigarrow b$ and $(a \cdot 1) \rightsquigarrow b$ are regular elements, we have $a \rightsquigarrow b=(a \cdot 1) \rightsquigarrow b$. Since $a \cdot 1=1 \cdot a$, we have $a \rightsquigarrow b=(1 \cdot a) \rightsquigarrow b$. Similarly, we can prove that $a \rightsquigarrow b=a \rightsquigarrow(1 \cdot b)=a \rightsquigarrow(b \cdot 1)$.

Proposition 26. Let $A=\langle A ; \cdot, \leq, 1\rangle$ be a bounded right quasi-ordered monoid. If $A$ is left residuated, then

(1) It has $\rightsquigarrow$ as the left residual of $\cdot$, which satisfies $a \cdot b \leq c \Leftrightarrow a \leq b \rightsquigarrow c$ and $(a \rightsquigarrow b) \cdot a \leq b$;

(2) $a \leq b \Leftrightarrow a \rightsquigarrow b=1$;

(3) If $a \leq b$, then $c \rightsquigarrow a \leq c \rightsquigarrow b$;

(4) If $a \leq b$ and $b \leq a$, then $1 \rightsquigarrow a=1 \rightsquigarrow b$;

(5) $a \rightsquigarrow b=(1 \cdot a) \rightsquigarrow b=(a \cdot 1) \rightsquigarrow b=a \rightsquigarrow(b \cdot 1)=a \rightsquigarrow(1 \cdot b)$.

If $A$ is right residuated, then

(6) It has $\rightarrow$ as the right residual of $\cdot$, which satisfies $a \cdot b \leq c \Rightarrow b \leq a \rightarrow c$ and $a \cdot(a \rightarrow b) \leq b$;

(7) $a \leq b \Leftrightarrow a \rightarrow b=1$;

(8) If $a \leq b$, then $b \rightarrow c \leq a \rightarrow c$ and $c \rightarrow a \leq c \rightarrow b$;

(9) If $a \leq b$ and $b \leq a$, then $1 \rightarrow a=1 \rightarrow b$;

(10) $a \rightarrow b=(a \cdot 1) \rightarrow b=(1 \cdot a) \rightarrow b=a \rightarrow(b \cdot 1)=a \rightarrow(1 \cdot b)$.

Proof. The proof is similar to Proposition 25.

Let $A=\langle A ; \cdot, \leq, 0,1\rangle$ be a bounded left (or right) quasi-ordered monoid with right residual. We can define the unary operation' on $1 \cdot A$ by $a^{\prime}=a \rightarrow 0$. Then we extend the operation on $A$ which need satisfy $a^{\prime} \cdot 1=(a \cdot 1)^{\prime}$. Moreover, it seems easily to see that $a^{\prime \prime}=a$ on $1 \cdot A$. Similarly, if $A=\langle A ; \cdot, \leq, 0,1\rangle$ is a bounded left (or right) quasi-ordered monoid with left residual, then we can define the unary operation $\urcorner$ on $1 \cdot A$ by $a\urcorner=a \rightsquigarrow 0$ and extend the operation on $A$ which need satisfy $a\urcorner \cdot 1=(a \cdot 1)\urcorner$.

Theorem 4. Let $A=\left\langle A ; \cdot \cdot 1, \cdot 2, \leq_{1}, \leq_{2}, 0,1\right\rangle$ be an algebra of type $\langle 2,2,2,2,0,0\rangle$ such that $(1)\left\langle A ; \cdot{ }_{1}, \leq_{1}, 0,1\right\rangle$ is a bounded left quasi-ordered monoid with $\cdot 1$ having right residual, (2) $\left\langle A ;{ }_{2}, \leq_{2}, 0,1\right\rangle$ is a bounded right quasi-ordered monoid with ${ }_{2}$ having left residual. Suppose that the unary operations and the residuals satisfy the supplementary conditions: 
(A) $\left.a^{\prime}=a\right\urcorner$;

(wPQW3) $(a \rightarrow b) \rightsquigarrow b=(b \rightarrow a) \rightsquigarrow a,(a \rightsquigarrow b) \rightarrow b=(b \rightsquigarrow a) \rightarrow a$;

(wPQW8) $a^{\prime \prime}=a$;

$($ wPQW9) $a \rightarrow(b \rightsquigarrow c)=b \rightsquigarrow(a \rightarrow c)$.

Then $\langle A ; \rightarrow, \rightsquigarrow,, 1\rangle$ is a weak PQW-algebra.

Proof. To prove that $\left\langle A ; \rightarrow, \rightsquigarrow,{ }^{\prime}, 1\right\rangle$ is a weak PQW-algebra, we need to show that (wPQW1), (wPQW2), (wPQW4), (wPQW5) and (wPQW7) hold.

(wPQW1) Since $(1 \rightarrow a) \rightsquigarrow a=(a \rightarrow 1) \rightsquigarrow 1=1$, we have $1 \rightarrow a \leq_{2} a$. Meanwhile, because $a \rightsquigarrow(1 \rightarrow a)=1 \rightarrow(a \rightsquigarrow a)=1 \rightarrow 1=1$, we have $a \leq_{2} 1 \rightarrow a$, so $1 \rightsquigarrow(1 \rightarrow a)=1 \rightsquigarrow a$. Similarly, $1 \rightarrow(1 \rightsquigarrow a)=1 \rightarrow a$. By (wPQW9), we have $1 \rightsquigarrow(1 \rightarrow a)=1 \rightarrow(1 \rightsquigarrow a)$, so $1 \rightarrow a=1 \rightsquigarrow a$.

(wPQW2) First we show that $(a \cdot 1 b) \rightarrow c=b \rightarrow(a \rightarrow c)$ and $(a \cdot 2 b) \rightsquigarrow c=a \rightsquigarrow(b \rightsquigarrow c)$. We calculate that $a \leq_{1}\left(a \cdot \cdot_{1} b\right) \rightarrow c$ iff $\left(a \cdot \cdot_{1} b\right) \cdot \cdot_{1} a \leq_{1} c$ iff $a \cdot \cdot_{1}\left(b \cdot \cdot_{1} a\right) \leq_{1} c$ iff $b \cdot \cdot_{1} a \leq_{1} a \rightarrow c$ iff $a \leq_{1} b \rightarrow(a \rightarrow c)$, thus $\left(a \cdot{ }_{1} b\right) \rightarrow c=b \rightarrow(a \rightarrow c)$. The other can be proved similarly. Hence $1 \rightarrow(a \rightarrow b)=\left(a \cdot{ }_{1} 1\right) \rightarrow b=a \rightarrow b$ and $1 \rightsquigarrow(a \rightsquigarrow b)=(1 \cdot 2 a) \rightsquigarrow b=a \rightsquigarrow b$.

(wPQW4) Since $b \rightarrow((b \rightarrow c) \rightsquigarrow c)=(b \rightarrow c) \rightsquigarrow(b \rightarrow c)=1$, we have $b \leq_{1}(b \rightarrow c) \rightsquigarrow c$, it follows that $a \rightarrow b \leq_{1} a \rightarrow((b \rightarrow c) \rightsquigarrow c)=(b \rightarrow c) \rightsquigarrow(a \rightarrow c)$, so $(a \rightarrow b) \rightarrow((b \rightarrow c) \rightsquigarrow(a \rightarrow c))=1$. The other can be proved similarly.

(wPQW5) We have $a^{\prime} \rightsquigarrow b^{\prime}=\left(a^{\prime} \cdot 21\right) \rightsquigarrow\left(b^{\prime} \cdot 21\right)=(a \rightsquigarrow 0) \rightsquigarrow(b \rightsquigarrow 0)=(a \rightarrow 0) \rightsquigarrow(b \rightarrow$ $0)=b \rightarrow((a \rightarrow 0) \rightsquigarrow 0)=b \rightarrow((0 \rightarrow a) \rightsquigarrow a)=b \rightarrow(1 \rightsquigarrow a)=1 \rightsquigarrow(b \rightarrow a)=b \rightarrow a$, so $\left(a^{\prime} \leadsto b^{\prime}\right) \rightarrow(b \rightarrow a)=1$. The other can be proved similarly.

(wPQW7) We have $1 \rightarrow(1 \rightarrow a)^{\prime}=1 \rightarrow((1 \rightarrow a) \rightarrow 0)=(1 \rightarrow a) \rightarrow 0=(1 \rightarrow a)^{\prime}$.

Theorem 5. Let $\langle A ; \cdot, \leq, 0,1\rangle$ be a bounded left quasi-ordered monoid with right residual and left residual. Suppose that the unary operations are defined as above and the residuals satisfy the supplementary conditions:

(A) $\left.a^{\prime}=a\right\urcorner$;

(wPQW3) $(a \rightarrow b) \rightsquigarrow b=(b \rightarrow a) \rightsquigarrow a,(a \rightsquigarrow b) \rightarrow b=(b \rightsquigarrow a) \rightarrow a$;

$($ wwPQW4) $(a \rightarrow b) \rightarrow((b \rightarrow c) \rightsquigarrow(a \rightarrow c))=1$;

(wPQW8) $a^{\prime \prime}=a$;

(wPQW9) $a \rightarrow(b \rightsquigarrow c)=b \rightsquigarrow(a \rightarrow c)$.

Then $\langle A ; \rightarrow, \rightsquigarrow, ', 1\rangle$ is a weak PQW-algebra.

Proof. We only check the conditions (wPQW1), (wPQW2) and (wPQW4). The (wPQW5) and (wPQW7) can be proved similarly as Theorem 4 .

For (wPQW1), we have $(1 \rightsquigarrow a) \rightarrow a=(a \rightsquigarrow 1) \rightarrow 1=1$ by (wPQW3) and Proposition 25, it follows that $1 \rightsquigarrow a \leq a$. Since $a \cdot 1 \leq a$, we have $a \leq 1 \rightsquigarrow a$ by the left residuated property. Thus $1 \rightsquigarrow a=(1 \rightsquigarrow a) \cdot 1=a \cdot 1$. Similarly, $1 \rightarrow a=1 \cdot a$. Hence we have $1 \rightarrow a=1 \rightsquigarrow a$.

For (wPQW2), we have $[1 \rightarrow(a \rightarrow b)] \rightsquigarrow(a \rightarrow b)=[(a \rightarrow b) \rightarrow 1] \rightsquigarrow 1=1$ by (wPQW3) and Proposition 26, thus $1 \rightarrow(a \rightarrow b) \leq a \rightarrow b$. On the other hand, $1 \cdot(a \rightarrow b) \leq a \rightarrow b$, we have $a \rightarrow b \leq 1 \rightarrow(a \rightarrow b)$. Thus $1 \cdot(a \rightarrow b)=1 \cdot[1 \rightarrow(a \rightarrow b)]$, which means that $1 \rightarrow(a \rightarrow b)=a \rightarrow b$. Similarly, $1 \rightsquigarrow(a \rightsquigarrow b)=a \rightsquigarrow b$ can be proved.

For (wPQW4), it is equivalent to $a \rightsquigarrow b \leq(b \rightsquigarrow c) \rightarrow(a \rightsquigarrow c)$, which by the right residuated property is equivalent to $(b \rightsquigarrow c) \cdot(a \rightsquigarrow b) \leq a \rightsquigarrow c$. To do this, we need show $(b \rightsquigarrow c) \cdot(a \rightsquigarrow b) \cdot a \leq c$ by the left residuated property. Using the monotony of $\cdot$, we have $(b \rightsquigarrow c) \cdot(a \rightsquigarrow b) \cdot a \leq(b \rightsquigarrow c) \cdot b \leq c$, the result is obtained. Adding the condition (wwPQW4), we have that (wPQW4) is true. 
Dually, we have the following result.

Theorem 6. Let $A=\langle A ; \cdot, \leq, 0,1\rangle$ be a bounded right quasi-ordered monoid with right residual and left residual. Suppose that the unary operation and the residuals satisfy the supplementary conditions:

(A) $\left.a^{\prime}=a\right\urcorner$;

(wPQW3) $(a \rightarrow b) \rightsquigarrow b=(b \rightarrow a) \rightsquigarrow a,(a \rightsquigarrow b) \rightarrow b=(b \rightsquigarrow a) \rightarrow a$;

$($ wwPQW4) $(a \rightsquigarrow b) \rightsquigarrow((b \rightsquigarrow c) \rightarrow(a \rightsquigarrow c))=1$;

(wPQW8) $a^{\prime \prime}=a$;

(wPQW9) $a \rightarrow(b \rightsquigarrow c)=b \rightsquigarrow(a \rightarrow c)$.

Then $\langle A ; \rightarrow, \rightsquigarrow, ', 1\rangle$ is a weak PQW-algebra.

Proof. It is similar to Theorem 5.

In the end, we need to point out that when $A$ is a left quasi-ordered monoid and a right quasi-ordered monoid, the condition (wwPQW4) in Theorem 5 or in Theorem 6 can be omitted, because it may be implied by other conditions. Hence we have the following result.

Corollary 1. Let $A=\langle A ; \cdot, \leq, 0,1\rangle$ be a bounded quasi-ordered monoid with left residual and right residual. Suppose that the unary operations and the residuals satisfy the supplementary conditions:

(A) $\left.a^{\prime}=a\right\urcorner$;

(wPQW3) $(a \rightarrow b) \rightsquigarrow b=(b \rightarrow a) \rightsquigarrow a,(a \rightsquigarrow b) \rightarrow b=(b \rightsquigarrow a) \rightarrow a$;

(wPQW8) $a^{\prime \prime}=a$;

(wPQW9) $a \rightarrow(b \rightsquigarrow c)=b \rightsquigarrow(a \rightarrow c)$.

Then $\left\langle A ; \rightarrow, \rightsquigarrow,{ }^{\prime}, 1\right\rangle$ is a weak PQW-algebra.

\section{Conclusions}

In this paper we continued the investigation on weak pseudo-quasi-Wajsberg algebras (weak PQW-algebras, for short). We introduced the notions of type-I filters, type-II filters, left weak filters and right weak filters of a weak PQW-algebra. We discussed some properties of them and showed the relationships between them. Moreover, we tried to discuss the connection between normal filters and filter congruences on a weak PQW-algebra. Finally, we showed the relations between weak PQW-algebras and bounded left (or right) quasi-ordered monoids. We proved that any weak PQW-algebra is a bounded left (or right) quasi-ordered monoid with right residual (or left residual). Conversely, if a bounded left (or right) quasi-ordered monoid with right residual and left residual and the residuals satisfy some supplementary conditions, then it is a weak PQW-algebra. In the future work, we will focus on the constructions of weak PQW-algebras and explore the applications in soft computing, fuzzy logics and quantum computational logics and so on.

Author Contributions: W.C. initiated the research and revised the paper. W.L. wrote the draft.

Funding: This research was funded by the National Natural Science Foundation of China (Grant No. 11501245), China Postdoctoral Science Foundation (No. 2017M622177) and Shandong Province Postdoctoral Innovation Projects of Special Funds (No. 201702005).

Conflicts of Interest: The authors declare no conflict of interest.

\section{References}

1. Chang, C.C. A new proof of the completeness of Lukasiewicz axioms. Trans. Am. Soc. 1959, 93, 74-80. 
2. Hajek, P. Metamathematics of Fuzzy Logic; Kluwer Academic Publishers: Dordrecht, The Netherlands, 1998.

3. Esteva, F.; Godo, L. Monoidal $t$-norm based logic: Towards a logic for left-continuous $t$-norms. Fuzzy Sets Syst. 2001, 124, 271-288. [CrossRef]

4. Iséki, K.; Tanaka, S. An introduction to the theory of BCK-algebras. Math. Jpn. 1978, 23, 1-26.

5. Jun, Y.B.; Roh, E.H. On the BCI-G part of BCI-algebras. Math. Jpn. 1993, 38, 697-702.

6. Di Nola, A.; Georgescu, G.; Iorgulescu, A. Pseudo BL-algebras: Part I. Mult. Valued Log. 2002, 8, 673-714.

7. Di Nola, A.; Georgescu, G.; Iorgulescu, A. Pseudo BL-algebras: Part II. Mult. Valued Log. 2002, 8, 715-750.

8. Dudek, W.A.; Jun, Y.B. Pseudo-BCI algebras. East Asian Math. J. 2008, 24, 187-190.

9. Georgescu, G.; Iorgulescu, A. Pseudo-BCK algebras: An extension of BCK algebras. In Combinatorics, Computability and Logic; Calude, C.S., Dinneen, M.J., Sburlan, S., Eds.; Springer: London, UK, 2001; pp. 97-114.

10. Georgescu G.; Iorgulescu, A. Pseudo MV algebras. Mult. Valued Log. 2001, 6, 95-135.

11. Hajek, P. Observations on non-commutative fuzzy logic. Soft Comput. 2003, 8, 38-43. [CrossRef]

12. Font, J.M.; Rodriguez, A.J.; Torrens, A. Wajsberg algebras. Stochastica 1984, VIII, 5-31.

13. Ceterchi, R. Pseudo-Wajsberg algebras. Mult. Valued Log. 2001, 6, 67-88.

14. Ceterchi, R. Weak pseudo-Wajsberg and weak pseudo-MV algebras. Soft Comput. 2001, 5, 334-346. [CrossRef]

15. Gudder, S. Quantum computational logic. Int. J. Theor. Phys. 2003, 42, 39-47. [CrossRef]

16. Ledda, A.; Konig, M.; Paoli, F.; Giuntini, R. MV algebras and quantum computation. Stud. Log. 2006, 82, 245-270. [CrossRef]

17. Chen, W.J.; Davvaz, B. Some classes of quasi-pseudo-MV algebras. Log. J. IGPL 2016, 24, 655-673. [CrossRef]

18. Liu, J.M.; Chen, W.J. A non-communicative generalization of quasi-MV algebras. In Proceedings of the 2016 IEEE International Conference on Fuzzy Systems, FUZZ-IEEE 2016, Vancouver, BC, Canada, 24-29 July 2016; pp. 122-126.

19. Liu, W.J.; Chen, W.J. Weak pseudo-quasi-Wajsberg algebras. In Proceedings of the 4th International Conference on Quantitative Logic and Soft Computing, QLSC 2016, Hangzhou, China, 14-17 October 2016; pp. $385-395$.

20. Bou, F.; Paoli, F.; Ledda, A.; Giuntini, R. The logic of quasi-MV algebras. J. Log. Comput. 2010, 20, 619-643. [CrossRef]

21. Burris, S.; Sankappanavar, H.P. A Course in Universal Algebra; The Millennium Edition; Springer-Verlag: Berlin, Germany, 1981.

22. Chen, W.J.; Dudek, W.A. Ideals and congruences in quasi-pseudo-MV algebras. Soft Comput. 2018, 22, 3879-3889. [CrossRef]

23. Zhang, X.H. Fuzzy anti-grouped filters and fuzzy normal filters in pseudo-BCI algebras. J. Intell. Fuzzy Syst. 2017, 33, 1767-1774. [CrossRef]

24. Chen, W.J. The new generalization of Wajsberg algebras. J. Mult. Valued Log. Soft Comput. 2017, 29, $135-156$.

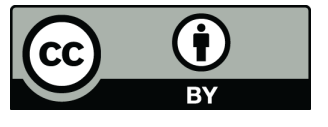

(C) 2019 by the authors. Licensee MDPI, Basel, Switzerland. This article is an open access article distributed under the terms and conditions of the Creative Commons Attribution (CC BY) license (http://creativecommons.org/licenses/by/4.0/). 